ARTICLE

https://doi.org/10.1038/s41467-018-07714-9

\title{
OPEN
}

\section{Regulation of mycobacterial infection by macrophage Gch1 and tetrahydrobiopterin}

\author{
Eileen McNeill (1) 1,2, Elena Stylianou (10 3, Mark J. Crabtree (10 1,2, Rachel Harrington-Kandt ${ }^{3}$, Anna-Lena Kolb ${ }^{1,2}$, \\ Marina Diotallevi, ${ }^{1,2}$, Ashley B. Hale ${ }^{1,2}$, Paulo Bettencourt ${ }^{3}$, Rachel Tanner ${ }^{3}$, Matthew K. O'Shea ${ }^{3}$, \\ Magali Matsumiya ${ }^{3}$, Helen Lockstone ${ }^{2}$, Julius Müller (10 ${ }^{3}$, Helen A. Fletcher (1) ${ }^{4}$, David R. Greaves ${ }^{5}$, \\ Helen McShane ${ }^{3} \&$ Keith M. Channon ${ }^{1,2}$
}

Inducible nitric oxide synthase (iNOS) plays a crucial role in controlling growth of $\mathrm{Myco-}$ bacterium tuberculosis (M.tb), presumably via nitric oxide (NO) mediated killing. Here we show that leukocyte-specific deficiency of NO production, through targeted loss of the iNOS cofactor tetrahydrobiopterin (BH4), results in enhanced control of M.tb infection; by contrast, loss of iNOS renders mice susceptible to M.tb. By comparing two complementary NO-

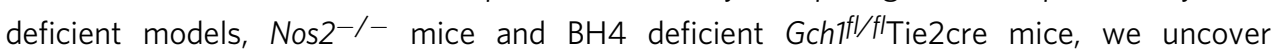
NO-independent mechanisms of anti-mycobacterial immunity. In both murine and human leukocytes, decreased Gch1 expression correlates with enhanced cell-intrinsic control of mycobacterial infection in vitro. Gene expression analysis reveals that Gch1 deficient macrophages have altered inflammatory response, lysosomal function, cell survival and cellular metabolism, thereby enhancing the control of bacterial infection. Our data thus highlight the importance of the NO-independent functions of Nos2 and Gch1 in mycobacterial control.

\footnotetext{
${ }^{1}$ Division of Cardiovascular Medicine, Radcliffe Department of Medicine, John Radcliffe Hospital, University of Oxford, Oxford OX3 9DU, UK. ${ }^{2}$ Wellcome Trust Centre for Human Genetics, University of Oxford, Oxford OX3 7BN, UK. ${ }^{3}$ Jenner Institute, University of Oxford, Oxford OX3 7DQ, UK. ${ }^{4}$ Department of Immunology and Infection, London School of Hygiene and Tropical Medicine, Keppel Street, London WC1E 7HT, UK. ${ }^{5}$ Sir William Dunn School of Pathology, University of Oxford, Oxford OX1 3RE, UK. These authors contributed equally: Eileen McNeill, Elena Stylianou. These authors jointly supervised this work: Helen McShane, Keith M. Channon. Correspondence and requests for materials should be addressed to E.M. (email: eileen.mcneill@well.ox.ac.uk) or to K.M.C. (email: keith.channon@cardiov.ox.ac.uk)
} 
T uberculosis (TB) remains a significant global health problem that kills more people than any other infectious disease, with $>10$ million new cases and 1.8 million deaths annually ${ }^{1}$. Chronic HIV co-infection and the treatment of chronic inflammatory conditions such as rheumatoid arthritis with potent immune modulators such as anti-TNF biologics, has revealed latent Mycobacterium tuberculosis (M.tb) infection reactivation as another growing source of $\mathrm{TB}$ disease burden, compounded by the global rise of drug-resistant TB. Thus, the discovery of new treatments for TB remains a pressing priority. In particular, augmenting host anti-mycobacterial mechanisms to enhance pathogen control through host-directed therapies (HDTs) is an area of intense interest. Identification of novel innate pathways and mechanisms could yield important insights that would lead to new therapeutic strategies.

Progress in the identification of genetic factors involved in TB susceptibility has been limited but the importance of some genes is already clear ${ }^{2}$. Polymorphisms in inducible nitric oxide synthase (iNOS, encoded by NOS2) ${ }^{3}$ confer important differences in the control of mycobacterial infection. Mice deficient in iNOS show variability in the timing of loss of control of mycobacterial infection in experimental models, however, in all published studies to date Nos $2^{-1-}$ mice show increased susceptibility at later time points. Nos $2^{-/-}$mice succumb to infection with intravenous Mycobacterium bovis Bacillus Calmette Guerin (BCG) after 8-12 weeks, in contrast to wildtype mice that are able to control infection $^{4}$. Furthermore, iNOS-deficient mice infected with the more virulent M.tb Erdman succumb more rapidly than control mice in both intravenous and aerosol challenge experiments, even if early control of infection is maintained in short challenges ${ }^{5-7}$. Thus, iNOS is a pivotal regulator of susceptibility to M.tb infection in mice. These effects of iNOS have been associated with the production of nitric oxide (NO), largely because of in vitro correlations between the mycobacteriocidal activity of activated murine macrophages and the amount of reactive nitrogen intermediates (RNI) produced ${ }^{8,9}$ and the direct cytotoxicity of nitric oxide to mycobacteria ${ }^{10}$.

The concept that NO, and thus iNOS, acts only to kill mycobacteria in infected macrophages overlooks the more recent advances in the understanding of NO and NOS-dependent nitroso-redox signaling. NO can mediate reduced susceptibility to infection by modulating a range of host cell functions including induction of cell apoptosis or necrosis, control of phagosome maturation, effects on iron metabolism and signaling to the adaptive immune system ${ }^{11}$. Many of these functions are mediated by nitric oxide and RNI as signaling molecules, transducing their signal through protein modifications such as nitrosylation of cysteine residues or protein nitration. However, iNOS may also have nitric oxide-independent functions. The NOS cofactor tetrahydrobiopterin (BH4) is essential for NO production and stabilizes iNOS dimer formation ${ }^{12}$. Under BH4 replete conditions the NOS isoforms generate NO by oxidation of the amino acid Larginine by molecular oxygen, forming L-citrulline ${ }^{13}$. NOS enzymes function in an 'uncoupled' state in the absence of BH4, whereby L-arginine is not oxidized to NO and superoxide is produced by the reductase domain of the enzyme. Whilst most attention on NOS uncoupling has been focused on endothelial $\mathrm{NOS}^{14,15}$, we have demonstrated more recently that macrophage $\mathrm{BH} 4$ regulates production of reactive oxygen species, and impacts nitroso-redox dependent gene expression ${ }^{16}$. iNOS also demonstrates direct protein-protein interactions with a number of proteins including those with ribosomal and metabolic functions $^{17}$ indicating that the ability of this protein to influence cell biology is much wider than through NO-production alone.

The human biology of iNOS and NO in tuberculosis is controversial ${ }^{18-20}$. Although detection of functional iNOS in patients with inflammatory conditions such as tuberculosis has been documented $^{21,22}$, the ability of human macrophages to produce nitric oxide in vitro is less clear. However, an often overlooked aspect of human macrophage biology is their limited $\mathrm{BH} 4$ content ${ }^{23}$. How macrophage iNOS functions under conditions of limiting BH4 supply serves as a unique model of human infection, and alterations in disease processes under these conditions may reveal findings of particular relevance to human therapeutics in comparison to experimental models in wildtype mice where a fulminant NO response is seen.

$\mathrm{BH} 4$ is produced by a multistep pathway involving three enzymes; GTP cyclohydrolase-1 (GTPCH-1), pyrovoyl tetrahydropterin synthase (PTPS) and sepiapterin reductase (SR ${ }^{23}$ GTPCH-1, encoded by Gch1, is involved in the initial and ratelimiting step of $\mathrm{BH} 4$ synthesis $^{24}$. We have generated a conditional knockout mouse with Gch1 deletion in leukocytes, preventing BH4 biosynthesis ${ }^{25}$.

Here we demonstrate that mice deficient in NO production, through either loss of iNOS protein or the essential NOS co-factor BH4 show divergent phenotypes of susceptibility vs enhanced control of mycobacterial infection both in vivo and in vitro. Using genomic analysis, we identify a gene signature, unique to the loss of BH4 and distinct from the loss of iNOS protein, that is associated with enhanced control of mycobacterial infection. These results demonstrate that NO-independent mechanisms of bacterial control by iNOS and $\mathrm{BH} 4$ have pathological importance and therapeutic potential in human disease.

\section{Results}

iNOS $^{+} G c h 1^{f l f l} T_{T i e} 2 c r e$ macrophages do not produce NO. We generated matched litters of Gch $1^{f l f l} \mathrm{Tie} 2 \mathrm{cre}$ and Gch $1^{f l f l}$ mice (hereafter referred to as wildtype), and confirmed that $G c h 1^{f l f l}$ Tie2cre mice lack Gch1 expression in hematopoietic cells (Supplementary Figure 1a-c). In vitro infection of macrophages with BCG (Pasteur) alone showed minimal induction of Nos2, however in the presence of IFN $\gamma$ infection with BCG caused a robust induction of Nos 2 and accumulation of nitrite, indicating robust iNOS NO-producing activity (Fig. 1a). The specificity of nitrite accumulation for iNOS activity was confirmed by infection of Nos $2^{-1-}$ BMDM under identical conditions (Supplementary Figure 2). BCG/IFN $\gamma$ treatment caused increased cellular BH4 content in wildtype mice, with BH4 levels and GTPCH protein significantly reduced to near undetectable levels in the Gch $1^{f / f f}$ Tie2cre cells (Figure 1b, c). Loss of BH4 did not affect induction of iNOS protein (Fig. 1b), but prevented iNOS NOproducing activity measured as nitrite accumulation, arginine-citrulline conversion and direct detection of authentic NO generation by EPR (Fig. 1d-f). In order to test whether iNOS protein had other non-NO-producing roles in mycobacterial function the $\mathrm{BCG} / \mathrm{IFN} \gamma$ combination was used in all in vitro studies to cause robust iNOS expression. These data confirm that whilst iNOS protein is induced in macrophages infected with BCG, in the absence of the co-factor BH4 no nitric oxide is produced, providing a system to discriminate iNOS functions reliant specifically on nitric oxide production.

Whilst nitric oxide production was absent, production of ROS was significantly increased in Gch $1^{f / f} /$ Tie2cre macrophages both at baseline (1.6 fold) and following BCG infection (3.2 fold) when assessed by the production of ethidium from dihydroethidium (Fig. 1g). This indicates that the loss of $\mathrm{BH} 4$ causes alterations in cellular redox state in both the presence and absence of iNOS. Macrophages have multiple potential sources of ROS, including the phagocytic NADPH oxidase that mediate oxidative burst and mitochondrial metabolism. To assess more broadly how ROS production by infected macrophages was affected, the phagocytic oxidative burst was assessed by flow cytometry using the CellROX 

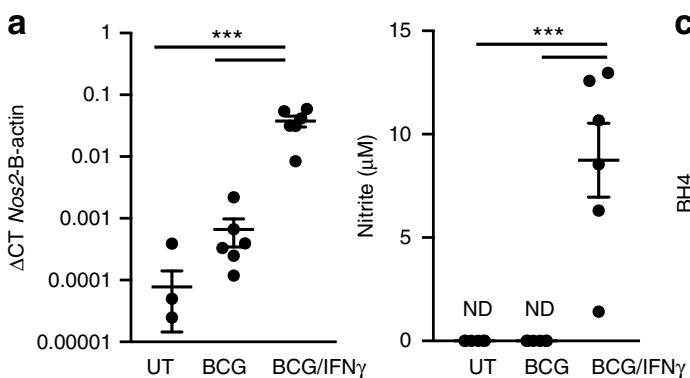
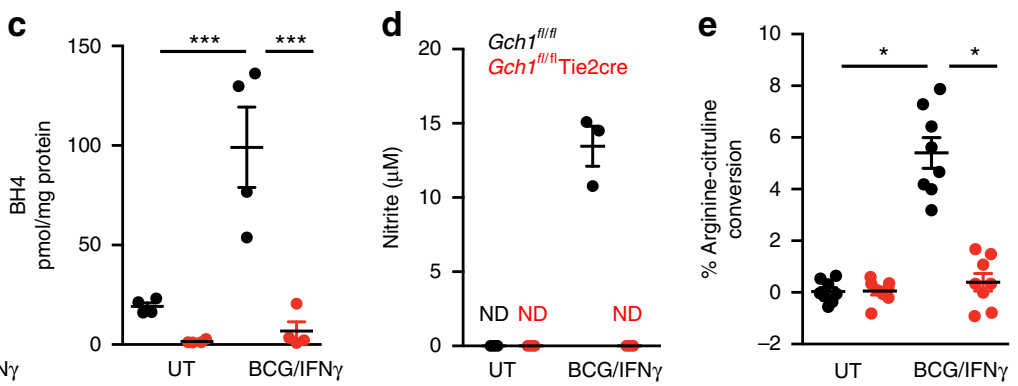

b

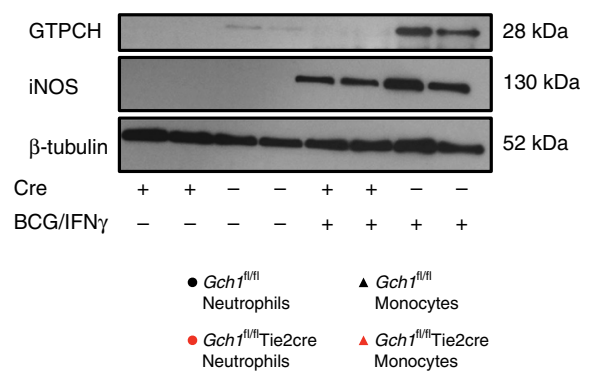

i

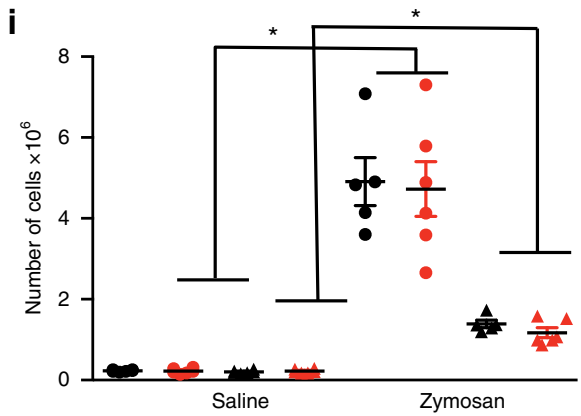

f

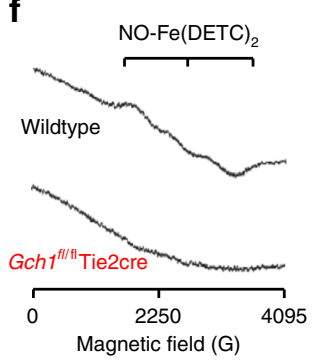

j

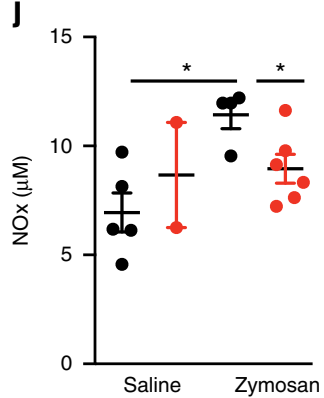

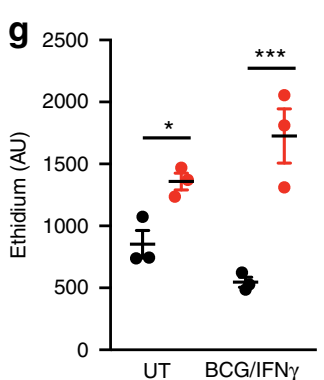

h

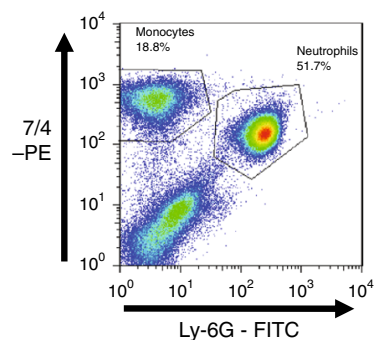

k

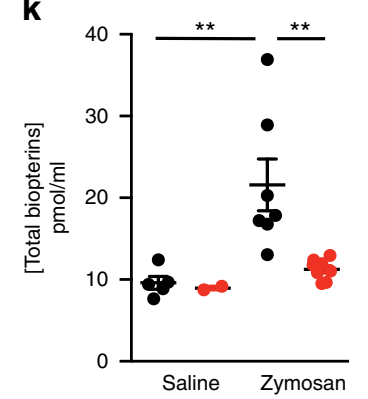

I

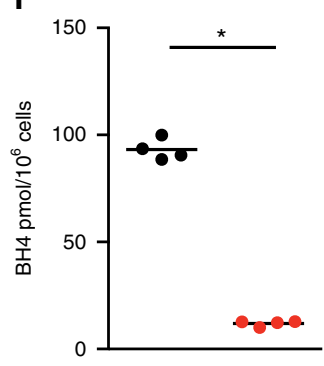

Fig. 1 Characterisation of the in vitro mycobacterial infection of Gch $f^{f / f l} / \mathrm{Tie} 2 \mathrm{cre}$ macrophages. a Measurement of Nos2 and nitrite accumulation in bone marrow-derived macrophages stimulated with BCG (MOI 1:1) or IFN $(10 \mathrm{ng} / \mathrm{ml})$ and BCG (MOI 1:1) for $24 \mathrm{~h}$ in wildtype macrophages $(n=6)$. b Western blotting showing GTPCH and iNOS protein expression in Gch $f^{f / / f l}$ Tie2cre macrophages, compared to wildtype (WT) macrophages at baseline and following infection with IFN $\gamma(10 \mathrm{ng} / \mathrm{ml})$ and BCG (MOI 1:1) for $24 \mathrm{~h}$. Equal protein loading was demonstrated by detection of $\beta$-tubulin. c Measurement of BH4

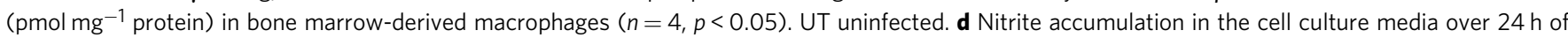
cell stimulation with BCG/IFN $\gamma$ as before was measured using the Griess assay ( $n=3 /$ genotype). ND not detectable. e Quantification of the NMAinhibitable arginine to citrulline conversion $(n=8, p<0.05)$. f Representative EPR spectra from $n=4$ /genotype WT and Gchfl/flTie2cre macrophages following activation with IFN $\gamma(10 \mathrm{ng} / \mathrm{ml})$ and BCG (MOI 1:1). $\mathbf{g}$ Cells were incubated with dihydroethidium for the last 30 min of culture and ethidium production was measured in cell lysates by HPLC ( $n=3$ /genotype). h Flow cytometry dotplot demonstrating identification of neutrophils ( $7 / 4^{\mathrm{HI}}$, Ly-6 $\mathrm{G}^{\mathrm{HI}}$ ) and inflammatory monocytes $\left(7 / 4^{\mathrm{HI}}, \mathrm{Ly}-6 \mathrm{G}^{-}\right)$in lavage fluid from wildtype animals $6 \mathrm{~h}$ after injection with $1 \mathrm{mg}$ zymosan ip. $\mathbf{i}$ The recruitment of monocytes and neutrophils by zymosan or saline control injection into wildtype and Gch fl/flTie2cre animals was quantified by flow cytometry in the peritoneal lavage $6 \mathrm{~h}$ later ( $n=6$ control groups, $n=5 \mathrm{Gch} 7^{f / / f l}$ Zymosan, $n=6 \mathrm{Gch} f^{f / f l}$ Tie2cre Zymosan). Lavage fluid was further analysed for total nitrite

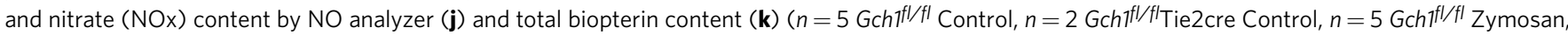

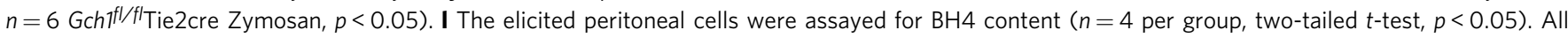
data analysed by one-way or two-way (genotype experiments) ANOVA with Bonferroni post-test unless otherwise stated above. All ' $n$ ' represent individual biological replicates, including cell cultures from individual animals/genotype. Data shown as individual data-points with mean and SEM. ${ }^{\star} p<0.05$, ${ }^{\star \star} p<0.01,{ }^{\star \star *} p<0.001$. Source blot and flow cytometry data are available in Supplementary Figure 10 and 11

indicator dye $\mathrm{e}^{26}$. BMDM were infected with BCG-GFP 27 and the ROS production was assessed in infected BCG-GFP+ vs uninfected BCG-GFP - cells $2 \mathrm{~h}$ after initial infection with BCG-GFP/IFN $\gamma$. Whilst a significant induction of ROS production was observed, this was not significantly affected by loss of BH4 (Supplementary Figure 3a). To further examine redox changes in response to infection we measured the extracellular release of $\mathrm{H}_{2} \mathrm{O}_{2}$, which is induced by infection and was undetectable in uninfected cells, this was significantly reduced in the Gch $1^{f l / f l}$ Tie2cre cells (Supplementary Figure 3b) indicating that the changes in redox biology elicited by the loss of $\mathrm{BH} 4$ in macrophages is substantially more complex than has been observed in other BH4 deficient cell types ${ }^{28}$. Whilst in endothelial cells ROS production is elevated as a result of eNOS uncoupling in the absence of $\mathrm{BH} 4$, in macrophages $\mathrm{BH} 4$ deficiency results in more widespread changes, but with the phagocytic oxidative burst in response to infection appearing unchanged.

Gch $1^{f l / f l}$ Tie2cre macrophages are BH4 and NO-deficient in vivo. To assess whether the loss of NO-production had biological consequences in vivo it was important to demonstrate that the cells remained deficient in both $\mathrm{BH} 4$ and $\mathrm{NO}$-production even in an environment where other cells were replete in BH4 levels. We confirmed Gch1 deletion rendered inflammatory cells $\mathrm{BH} 4$ deficient in vivo, by inducing sterile peritonitis in mice using 
zymosan, to induce iNOS in recruited peritoneal exudate cells ${ }^{29}$. Recruitment of neutrophils and monocytes to the peritoneal cavity (Fig. 1h) was unaffected by Gch1 deletion (Fig. 1i) ${ }^{25}$. Analysis of peritoneal lavage fluid in wildtype mice revealed that zymosan caused significant induction of the iNOS and tetrahydrobiopterin pathway, measured by total biopterins and nitrite/ nitrate levels (NOx), stable oxidized extracellular endproducts of nitric oxide production in vivo (Fig. 1j, k). Total biopterins (BH4, dihydrobiopterin and biopterin) rather than $\mathrm{BH} 4$ were assessed due to the oxidizing nature of extracellular environment causing oxidative loss of $\mathrm{BH} 4$ to the oxidized forms dihydrobiopterin and biopterin. In contrast, no significant elevation of biopterins or NOx levels were evident in peritoneal lavage fluid from Gch $1^{f l}$ $f l_{\text {Tie2cre mice. Furthermore, peritoneal leukocytes from } G c h 1^{f l}}$ $f l$ Tie2cre animals showed barely detectable levels of intracellular $\mathrm{BH} 4$, in contrast to wildtype cells (Fig. 11) indicating that Gch $1^{\mathrm{fl} /}$ ${ }^{f}$ Tie2cre leukocytes remain $\mathrm{BH} 4$ deficient in vivo and are not rescued by systemic $\mathrm{BH} 4^{28}$.

Gch $1^{f l f l}$ Tie2cre mice have enhanced control of BCG infection. Given the importance of iNOS in mycobacterial infection in mice, we next investigated the consequences of $\mathrm{BH} 4$ deficiency on mycobacterial infection in vivo, comparing wildtype with Gch $1^{f l}$ $f_{\text {Tie2 }}$ cre mice. We confirmed that peripheral blood leukocyte homeostasis was not altered in Gch1 flfft Tie2cre mice (Supplementary Figure $4 \mathrm{a}-\mathrm{d}$ ) and that Gch $1^{f l / f l}$ Tie2cre alveolar macrophages from bronchoalveolar lavage were deleted for Gch1 (Fig. 2a), and had undetectable BH4 levels (Fig. 2b). We infected wildtype and Gch $1^{f l / f l}$ Tie2cre mice with BCG by intranasal instillation, and monitored the infected mice for 4 weeks. Both wildtype and $G c h 1^{f l f f l}$ Tie2cre mice had comparable lung bacterial load, but CFU counts were significantly lower in the Gch $1^{f / f l}$ Tie2cre spleens compared to wildtype mice littermates (Fig. 2c, d). BCG infection induced a significant increase in spleen:body weight ratio in Gch $1^{f l / f l}$ Tie2cre mice, and a non-significant increase in wildtype animals (Fig. 2e). Measurement of total biopterins in the lung and spleen homogenate showed a significant increase in infected wildtype lungs compared to uninfected controls (Fig. 2f). Total biopterin levels rather than $\mathrm{BH} 4$ alone were assessed due to the oxidizing nature of the homogenisation process required for BCG CFU assessment. The levels of total biopterins in the lung and spleen of infected mice were significantly lower in the Gch $1^{f l / f l}$ Tie 2 cre mice than the wildtype controls (Fig. 2f, g). The total biopterin content of BCG-infected lungs was significantly correlated with lung mycobacterial load (Fig. 2h), consistent with BH4 deficiency resulting in control of infection. BCG infection induced iNOS in the infected lung tissues from mice of both genotypes (Fig. 2i), but NOx in lung lysates was significantly lower in infected Gch $1^{f l / f l}$ Tie2cre mice (Fig. 2j).

Loss of NO but not iNOS protein reduces $M . t b$ infection. To more stringently assess the role of macrophage nitric oxide production in a more pathogenic model we evaluated the effect of macrophage $\mathrm{BH} 4$ deficiency on the response to a virulent aerosolized M.tb challenge in Gch $1^{f l / f l}$ Tie2cre mice. To directly compare the effects of total iNOS deficiency with deficient iNOSmediated NO production, we also infected Nos $2^{-1-}$ and control C57BL/6J mice under the same conditions. Inclusion of this control group was of particular importance given the variability in onset of susceptibility seen in previous studies ${ }^{5-7}$. As expected, Nos $2^{-1-}$ mice developed dramatic macroscopic lung and spleen pathology after M.tb infection, with numerous large lung granulomatous lesions and splenomegaly (Fig. 3a and Supplementary Figure 5). In contrast, none of these features were prominent in either the $G c h 1^{f l} / f l$ Tie2cre or the wildtype animals. Both wildtype control mice and $G c h 1^{f l / f l}$ Tie2cre mice gained weight over 4 weeks after infection (Fig. 3b). In contrast, Nos $2^{-1-}$ mice showed a steady decline in weight from 2 weeks post-infection, and required termination of the experiment 23 days postinfection at a pre-specified humane endpoint. The Nos $2^{-1-}$ mice had significantly higher lung and spleen CFU counts than the C57BL/6J control group (Fig. 3c, d), whereas CFU counts in the Gch $1^{f l / f l}$ Tie2cre mice were not different from their wildtype littermate controls. Body weight correlated with lung CFU counts in individual animals (Fig. 3e), with $G c h 1^{f l / f l}$ Tie2cre mice having highest bodyweights, in keeping with the lowest lung CFU. Immunohistochemistry of lung granuloma confirmed iNOS expression and macrophage infiltration within the granulomata of wildtype and Gch $1^{f l f t}$ Tie2cre mice that was absent in Nos2 $2^{-1-}$ mice (Fig. 3a and Supplementary Figure 6).

To further assess the control of M.tb infection in Gch $1^{f l / f l}$ Tie2cre mice, we undertook more prolonged infection experiments. Six weeks after infection with aerosolized M.tb, Gch $1^{f l / f l}$ Tie2cre mice had significantly lower CFU per lung than their wildtype littermate controls (Fig. 3f). Spleen CFU counts were comparable between groups (Fig. 3g). Whilst the reduced extrapulmonary spread of infection, seen when mice were infected with BCG, was not maintained when $G c h 1^{f l / f l}$ Tie2cre mice are exposed to the more virulent $M . t b$ infection, this stringent test of susceptibility shows a clear reduced susceptibility in the lung at a later 6-week timepoint. As expected, Gch1 gene expression in the infected Gch $1^{f l / f l}$ Tie2cre tissues (Supplementary Figure 7) was significantly lower whereas expression of other key inflammatory genes, Nos2, $T n f \alpha$ and Cd68, were unaffected, indicating preservation of the inflammatory response.

Gene expression reveals signature unique to loss of Gch1. Due to the multiple changes in the redox biology of the $\mathrm{BH} 4$ deficient macrophages we next sought to use gene expression analysis to gain insight into the mechanism of the enhanced control of mycobacterial infection observed in Gch1-deleted, BH4deficient macrophages. We performed a mouse whole genome array analysis on Gch $1^{f l / f l}$ Tie 2 cre and Nos $2^{-l-}$ macrophages, infected with BCG in the presence of IFN $\gamma$ for $24 \mathrm{~h}$ (to induce robust iNOS expression), compared with control uninfected macrophages for each genotype. This experimental design enables us to specifically compare the conditions of loss of iNOS and loss of iNOS-mediated NO-production in the context of $\mathrm{BH} 4$ deficiency.

We identified differentially expressed genes in response to BCG/IFN $\gamma$ in wildtype mice. As expected, BCG/IFN $\gamma$ caused major changes in macrophage gene expression profiles, with 12,412 probes being differentially expressed (Supplementary Data 1). Heatmap analysis of the 1000 most regulated genes showed a consistent activation across all genotypes (Supplementary Figure 8). Pathway analysis of the most significantly regulated genes, using the Ingenuity Pathway Analysis (IPA) tool, indicated activation of pathways associated with inflammation and infection (Supplementary Figure 8 and Supplementary Data 2) as expected.

We next compared the effect of loss of Gch1 and Nos 2 in both BCG/IFN $\gamma$ treated and uninfected macrophages. We observed only minor variation in uninfected macrophages between Gch $1^{\mathrm{fl}}$ ${ }^{f} l_{\text {Tie }}$ cre and Nos $2^{-l-}$ macrophages and their controls, with only 77 and 26 probes, respectively, being differentially expressed (Fig. 4a and Supplementary Data 3). In contrast, when infected with BCG, 779 (Gch $1^{f l / f l}$ Tie2cre vs Gch1 $\left.1^{f l f l}\right)$ and $172\left(\right.$ Nos2 $2^{-l-}$ vs Nos $2^{+/+}$) probes showed differential expression (Fig. 4a and Supplementary Data 3), with 737 probes being unique to the gene 
a

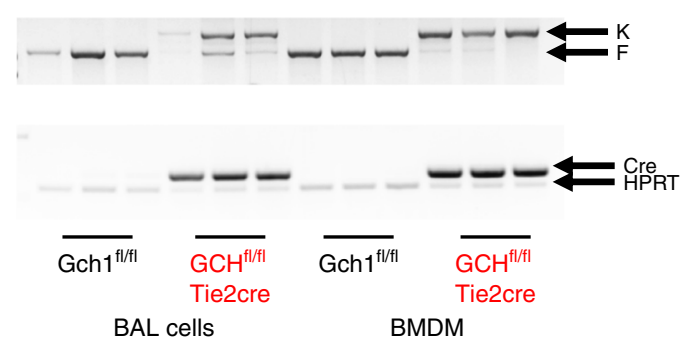

C

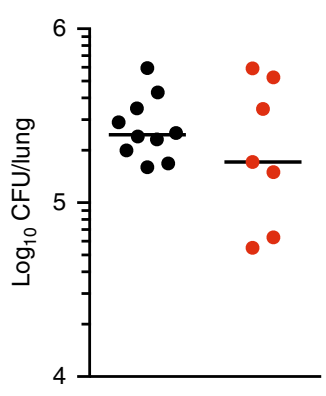

d

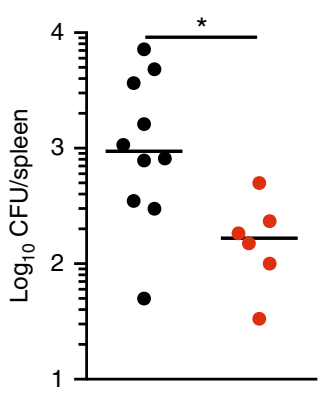

$\mathbf{f}$

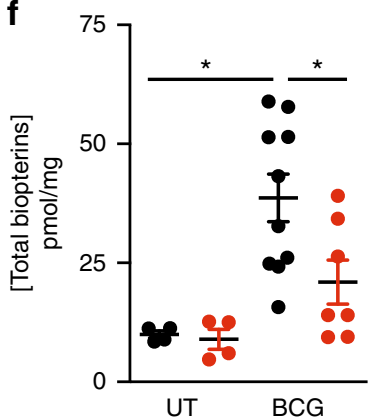

g

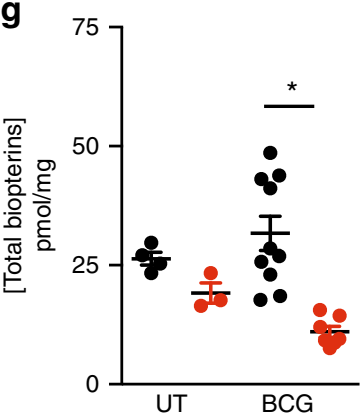

i

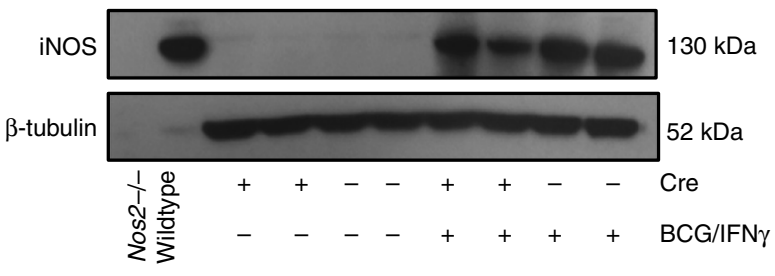

b

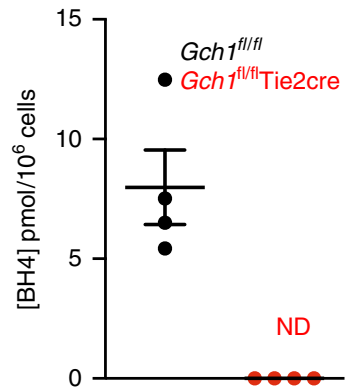

e

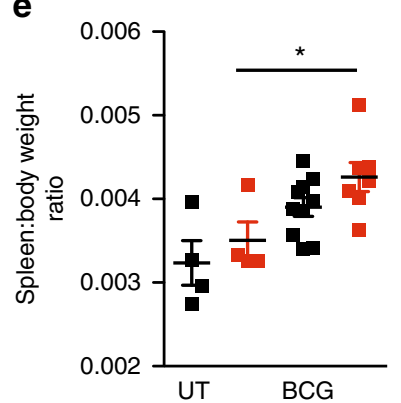

h

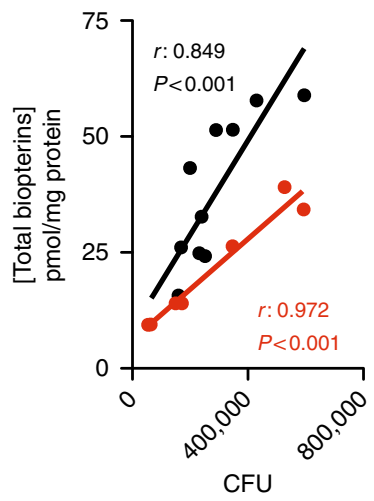

j

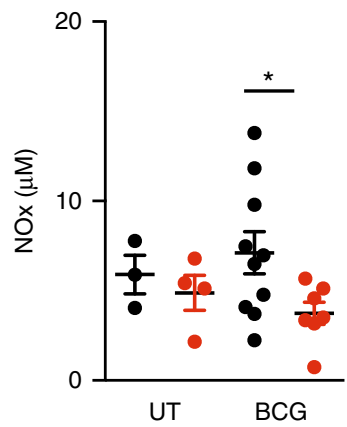

list for infected $G c h 1^{f l / f l}$ Tie2cre macrophages. IPA pathway analysis revealed significant activation, in Gch $1^{f l} / f l$ Tie2cre macrophages, of key functions relating to cell-cell signaling, inflammatory response and lipid metabolism (Fig. 4b, c). In particular, Gch1 deletion up-regulated pathways regulating myeloid cell recruitment, neutrophil activation, phagocyte degranulation, and sterol synthesis, whereas cell death and apoptosis of leukocytes were significantly down-regulated (Fig. 4b, c, Supplementary Data 4). Analysis of potential upstream regulators identified enhanced signaling via TLRs $2 / 4 / 5 / 9$ and inflammatory cytokine/ cytokine receptors including IL1, TNF superfamily, and CCL5 (Fig. 4d, Supplementary Data 5). These data indicated that part of the inflammatory response to infection appeared to be enhanced in the $\mathrm{BH} 4$ deficient macrophages. We validated the presence of genes that show regulation only as a result of $\mathrm{BH} 4$ deficiency $(A s 3 m t)$ and as a result of loss of both BH4 and iNOS (Slc40a1) in our gene array study in an independent cohort of cells (Fig. 5). These data confirmed the gene expression changes observed in our earlier study of LPS/IFN $\gamma$ stimulated macrophages, with the identification of similar gene expression changes in both As3mt and Slc40a1 ${ }^{25}$ and a similar prediction of decreased NRF2mediated gene expression (Fig. 4d). However, it also demonstrated striking divergence regulation of key genes such as IL-6 which showed decreased activation following LPS/IFN $\gamma$ treatment 
Fig. 2 Absence of Gch1 improves the resistance of mice to in vivo BCG infection. a The excision of the floxed Gch1 allele in both cells from WT and Gch $7^{f / f l}$ Tie2cre recovered by bronchial-alveolar lavage (BAL) was confirmed by genomic PCR, with analysis of BMDM used as a positive control for cre activity: knockout allele (K) floxed allele (F). The presence or absence of the cre transgene was confirmed using HPRT as a housekeeping gene. $\mathbf{b}$ The BH4 (pmol/106 cells) content of the BAL cells was analysed by HPLC ( $n=4$ per genotype, two-tailed $t$-test, $p<0.05)$. c, d Gch $f^{f l} / f l T i e 2 \mathrm{cre}($ red $)$ and wildtype (black) mice were infected intranasally with $5 \times 10^{6}$ colony forming units (CFU) BCG Pasteur. Four weeks following infection the lungs and spleen were removed and homogenised for analysis along with tissues from a matched cohort of uninfected animals. The bacterial load of the organs was enumerated

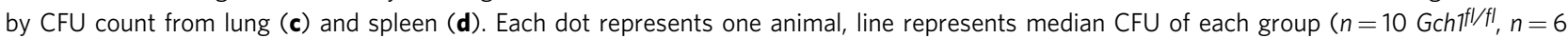
Gch $7^{f / f l}$ Tie2cre (Spleen), $n=7$ Gch $7^{f l / f l}$ Tie2cre (Lung) per group), $p<0.01$ (Mann-Whitney test). e Spleen to body weight ratio was also calculated.

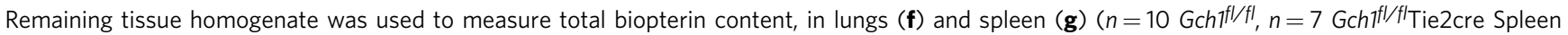
(infected) and $n=4$ /genotype (uninfected), $p<0.05$ ). Total lung biopterin content was correlated to lung CFU in all animals. Pearson $r p<0.05$ (h). i iNOS protein expression was confirmed by western blotting, with $B$-tubulin loading control ( $n=2$ per group shown, representative of $n=3$ independent biological replicates assayed in total). An NO analyzer was used to measure NOx content in the lung homogenates $(p<0.05)(\mathbf{j})$. All data analsyed with ANOVA with Bonferroni post-test (apart from (b-d) and (h) as above). Data shown as individual data-points with mean and SEM (apart from (c) and (d) as above). ${ }^{\star} p<0.05$. Source gel and blot images are available in Supplementary Figure 10

but enhanced expression following BCG/IFN $\gamma$ treatment (Fig. 4c), indicating exposure to a live pathogen causes a unique pattern of gene regulation that cannot be adequately modelled by the use of other proinflammatory stimuli.

To more broadly evaluate the entire dataset, we performed unsupervised Gene Set Enrichment Analysis (GSEA) to identify coordinated regulation of annotated groups of genes, implicated in defined cellular processes ${ }^{30}$. We performed Preranked GSEA using the GO term-defined gene sets relating to biological processes (MSigDB C5 BP) followed by network analysis of the most significantly altered gene sets using Enrichment Map ${ }^{30}$ (Fig. 6a, b, Supplementary Data 6). We then compared the lists of significantly altered gene sets for each genotype comparison to identify which were shared between genotypes and which were unique to the loss of Gch1 (Supplementary Figure 9a) these new gene set sub groups were again visualized using Enrichment Map (Fig. 6c and Supplementary Figure 9B). The majority of gene sets relating to DNA repair, protein catabolism, vesicular trafficking and ribosome activity were found to be common to the loss of either Gch1 or Nos2 (Supplementary Figure 9b). However, a number of gene signatures were unique to the loss of Gch1 (Fig. 6c, d). These gene sets were related to fundamental cellular processes such as metabolism and cellular detoxification as well as to processes related to inflammation and infection such as lysosomal function, apoptosis and cell senescence and innate immune. To confirm the biological relevance and validity of these findings, we performed a second round of GSEA using gene sets relating to canonical pathways and curated datasets (MSigDB C2 $\mathrm{CP})$. This analysis again identified a number of gene sets that were either shared by both genotypes or unique to the loss of Gch1 (Fig. 7 and Supplementary Data 6). Gch1 unique gene sets again included those related to lysosome function, inflammation, DNA damage response (including p53 pathway) and lipid and glucose metabolism confirming our findings.

Gch $1^{f l / f l}$ Tie2cre macrophages showed reduced IL-6 gene and protein expression in response to BCG/IFN $\gamma$, as predicted by the pathway analysis (Fig. 8). Under the conditions used for gene expression analysis no alterations in cell viability were seen (Fig. 9a). Under conditions of enhanced greater stress, with cells being infected with BCG/IFN $\gamma$ (MOI 5:1) we observed a decreased macrophage cell survival, associated with increased apoptosis of infected cells, in the absence of Gch1 again confirming that the gene expression analysis is predictive of alterations in macrophage cell biology. Gene sets related to the Sterol/Cholesterol pathway can have multiple effects on cell lipid handling including altered cholesterol handling and lipid droplet format, altered oxysterol production and alterations to LXR/RXR ligands. We tested the ability of $\mathrm{BH} 4$ deficient cells to form lipid droplets in response to $\mathrm{BCG} / \mathrm{IFN} \gamma$, using a recently reported method and droplet formation still occurred in the absence of $\mathrm{NO}$ (Fig. 9b $)^{31}$.

Gch1 expression alters mycobacterial growth in vitro. To determine whether Gch1 and BH4 directly alter control of mycobacterial infection in macrophages, we infected BMDM macrophages in vitro with BCG and quantified the control of mycobacterial infection using the mycobacterial growth inhibition assay (MGIA) ${ }^{32}$. After BCG infection, CFU counts were significantly higher in Nos $2^{-1-}$ macrophages than their wildtype controls (Fig. 10a). In contrast, Gch $1^{\text {flflfl }}$ Tie 2 cre macrophages had significantly lower CFU counts than their wildtype controls (Fig. 10b). This indicates that $\mathrm{BH} 4$-deficient macrophages alone have an enhanced cell-intrinsic mechanism for control of mycobacterial growth.

Having shown that Gch1 deletion increases the control mycobacterial infection by murine macrophages, we next tested whether a similar association between GCH1 expression and mycobacterial control is seen in human cells. PBMCs from BCGvaccinated infants, from a previously reported vaccine efficacy study $^{33}$, were used to perform an in vitro MGIA to assess control of BCG infection. In parallel, samples were infected with BCG overnight and underwent analysis for GCH1 and NOS2 gene expression. There was a significant positive correlation between GCH1 gene expression and increased mycobacterial CFU in the direct PBMC MGIA assay (Fig. 10c). In contrast, there was no correlation between NOS2 gene expression and mycobacterial control (Fig. 10d). Thus, lower GCH1 expression in human PBMCs is also associated with increased control of mycobacterial infection, independent of PBMC NOS2 expression.

\section{Discussion}

Our findings suggest that re-evaluation of the role, and mechanism of action, of NO in macrophage anti-mycobacterial activity is required. Along with other recent studies our data show that a direct cytotoxic role for NO within the macrophage is not the only, and possibly not the main, function for $\mathrm{NO}^{34,35}$. Whilst direct exposure of mycobacteria to nitric oxide gas has been confirmed to mediate mycobacterial killing ${ }^{10}$, our observations suggest that NO production by leukocyte-expressed iNOS is not the only mechanism by which iNOS can control M.tb and indeed a lack of NO maybe beneficial in some circumstances. A reinterpretation of the role of $\mathrm{NO}$ in $\mathrm{TB}$ has important implications for understanding the apparent discordance between the strong evidence for iNOS and NO in the control of mycobacteria in murine models, compared with a less clearly defined role for iNOS and NO system in human disease ${ }^{18-20}$. Although iNOS is expressed in inflammatory conditions such as $\mathrm{TB}^{21,22}$ the ability 
a
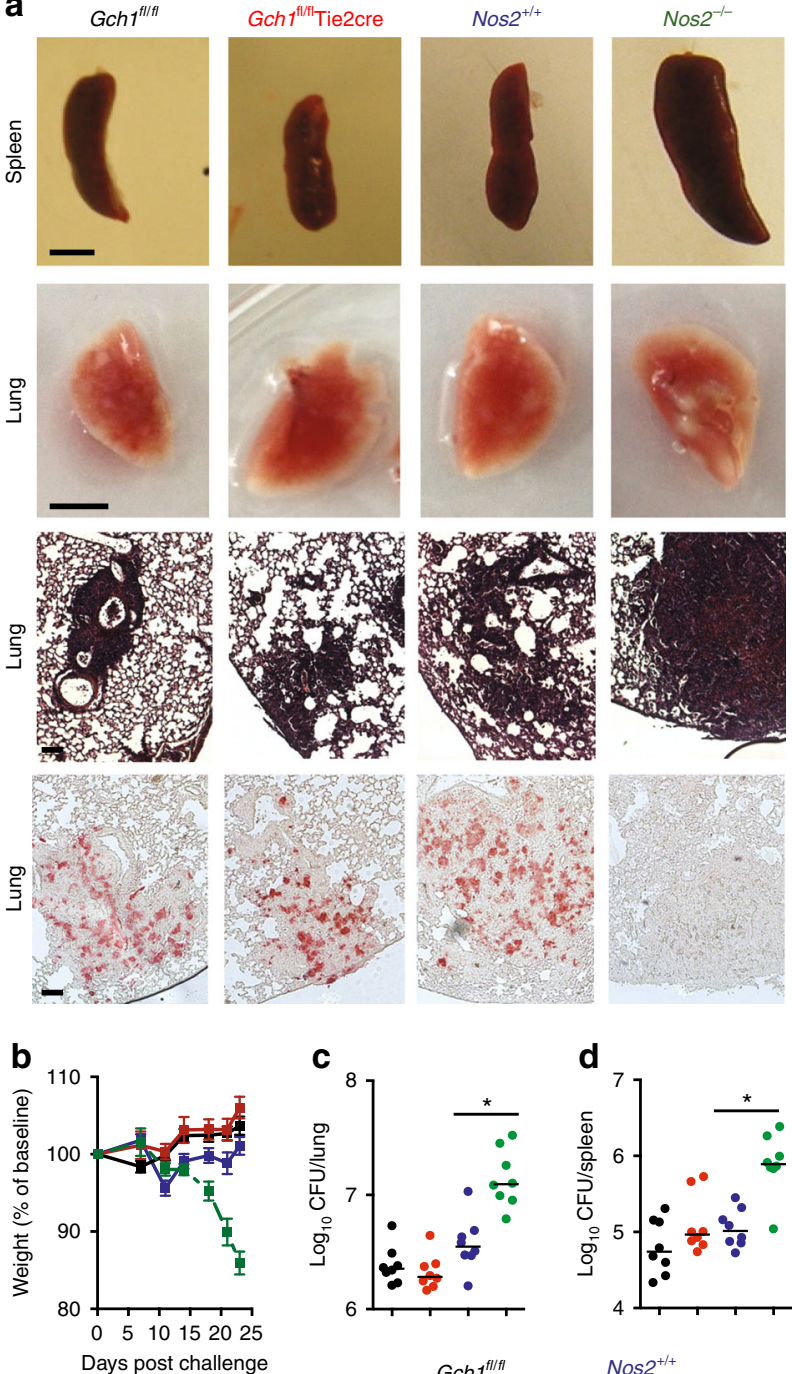

d

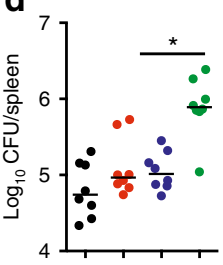

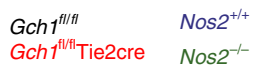

e
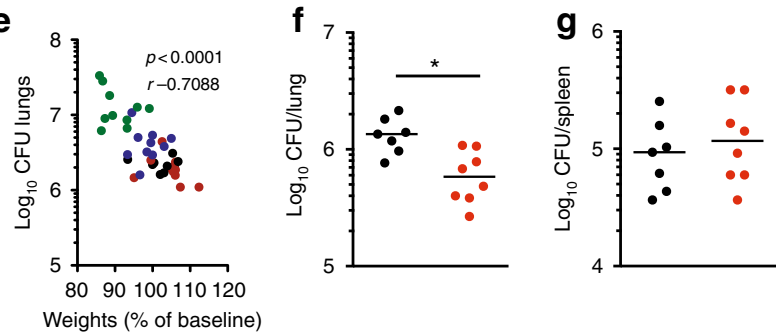

Weights (\% of baseline)

of human macrophages to produce nitric oxide is controversial ${ }^{36}$. SNPs in the NOS2 gene have been associated with altered susceptibility to disease, with many identified SNPs sitting in the promoter region and associated with increased gene transcription and associated with protection from $\mathrm{TB}^{3}$. These data do indicate that alterations in iNOS biology have an impact on human susceptibility, however our data would suggest that this may not be a simple association with $\mathrm{NO}$ cytotoxicity, but that the relative expression of NOS2 and GCH1 is likely to be key.

iNOS is expressed in macrophages from TB patients, but human macrophages exhibit exon skipping during transcription of 6-pyruvoyl tetrahydropterin synthase (PTPS), the second step in $\mathrm{BH} 4$ production, which leads to low-level production on $\mathrm{BH} 4$ in these cells, and accumulation of the proximal metabolite,
Fig. 3 Absence of Gch1 increases resistance of mice to virulent M.tb infection. Gch $7^{f l} / f l$ Tie2cre (red) and wildtype (black), Nos2 $2^{-/-}$(green) and Nos2 $2^{+/}$(blue) mice were infected with 150 CFU M.tb (Erdman strain) by aerosol inhalation. 23 days following infection the lungs and spleen were removed for analysis. Representative organs were imaged from each genotype prior to $n=2$ tissues per genotype being embedded for histochemical analysis. Sections were cut from all tissues and stained with H\&E or subjected to anti-iNOS immunohistochemistry (a). Animals were weighed at regular intervals throughout the experiment and the mean weight change and SEM per group was plotted (b). Lungs and spleen were homogenised and the mycobacterial load of the organs was enumerated by CFU count from lung (c) and spleen (d) ( $n=8$ per group, * $p<0.01)$ (Mann-Whitney). Each dot represents one mouse, line in each group represents the median CFU. e Weights negatively correlated with CFU counts. Pearson $r p<0.05$. f Gch $f^{f / f f}$ Tie2cre (red) and Gch $f^{f l / f l}$ (black) mice were infected as before but this time lungs and spleen were removed 6 weeks following infection for analysis. Lungs and spleen were homogenised and the mycobacterial load of the organs was enumerated by CFU count from lung (f) and spleen $(\mathbf{g}) n=7 \mathrm{Gch}$ fl/fl, $^{\mathrm{f}}=8 \mathrm{Gch} 7^{f / / f l} \mathrm{Tie} 2 \mathrm{cre}$, ${ }^{*} p<0.01$ (Mann-Whitney). The finding in (f) was replicated on two occasions in independent experiments. Scale bars: upper panels $0.5 \mathrm{~cm}$, lower panels 100 um

neopterin, that has been used as a biomarker in $\mathrm{TB}^{37}$. The $\mathrm{GCH1}$ gene has been identified as a marker of active disease in TB patients in several gene expression studies ${ }^{38,39}$. However, these studies did not report any further association other than increased expression in infected individuals, compared to healthy or disease control patients. In contrast, we now demonstrate that GCH1 expression, rather than NOS2 expression, correlates with control of mycobacterial growth in human PBMC. Indeed, a previous study has reported increased mycobacterial growth in infected human macrophages in the presence of exogenous BH4 supplementation ${ }^{40}$. Thus, human macrophages may share the NO-independent means of controlling mycobacteria under conditions of low $\mathrm{BH} 4$ availability, suggesting that inhibition of macrophage $\mathrm{BH} 4$ synthesis may provide a therapeutic target for TB.

INOS is increasingly recognized as having a signaling role in TB by preventing excessive inflammation and tissue damage. A recent study identified HIF-1a, downstream of iNOS activation, as a crucial mediator of IFN $\gamma$ mediated response to M.tb infection $^{35,41}$. Nos $2^{-1-}$ mice were used to demonstrate that iNOS was a crucial stabilizer of HIF-1 $\alpha$, resulting in increased HIF-1 $\alpha$ signaling ${ }^{41}$. Genetic deletion of either iNOS or HIF-1 1 resulted in reduced control of mycobacteria by macrophages. Critically iNOS and HIF-1 $\alpha$ mediated opposing roles in the regulation of a panel of pro-inflammatory cytokines, including IL-6, IL-1 and neutrophil chemokines, indicating iNOS has wider roles in the cell signaling than via HIF- $1 \alpha^{41}$. The ability of NO to chemically modify proteins through nitrosylation of cysteine residues is one way in which iNOS can transduce signals within the cell. In the case of mycobacterial infection, nitrosylation of NLRP3 prevents inflammasome activation thus reducing the production of important pro-inflammatory mediators such as IL- ${ }^{42}$. Accordingly IL-1 and lipoxygenase mediated pathways were shown to be overactive in $N o s 2^{-9-}$ mice with the resultant excessive neutrophil recruitment and tissue damage supporting enhanced mycobacterial growth ${ }^{34}$. BH4 deficient NO-deficient macrophages, appear to show transcriptional changes in similar gene sets and molecules highlighted in earlier studies in $\mathrm{Nos}^{-1-}$ cells and tissues ${ }^{34,41}$. Intriguingly, cellular pathways identified through analysis of iNOS protein-protein interaction ${ }^{17}$, namely ribosomal and proteosomal pathways, are found amongst the shared 
a

\begin{tabular}{|c|c|c|}
\hline & UT & $\mathrm{BCG} / \mathrm{IFN} \gamma$ \\
\hline 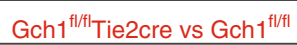 & 77 & 779 \\
\hline Nos2 $2^{-/-}$vs Nos $2^{+/+}$ & 26 & 172 \\
\hline
\end{tabular}

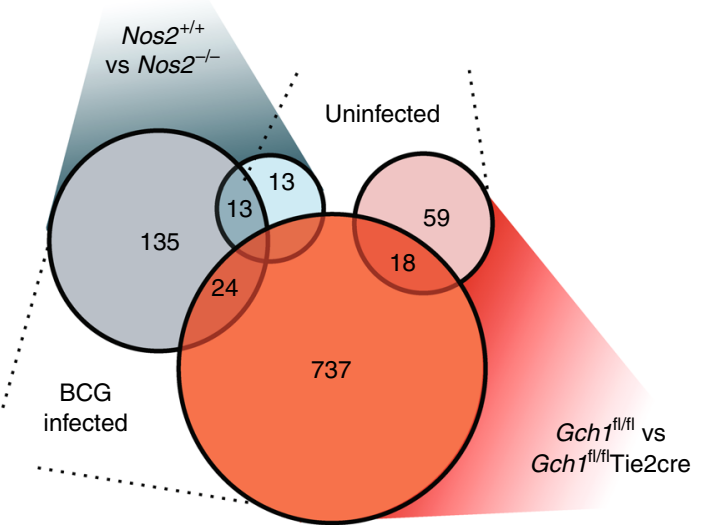

C

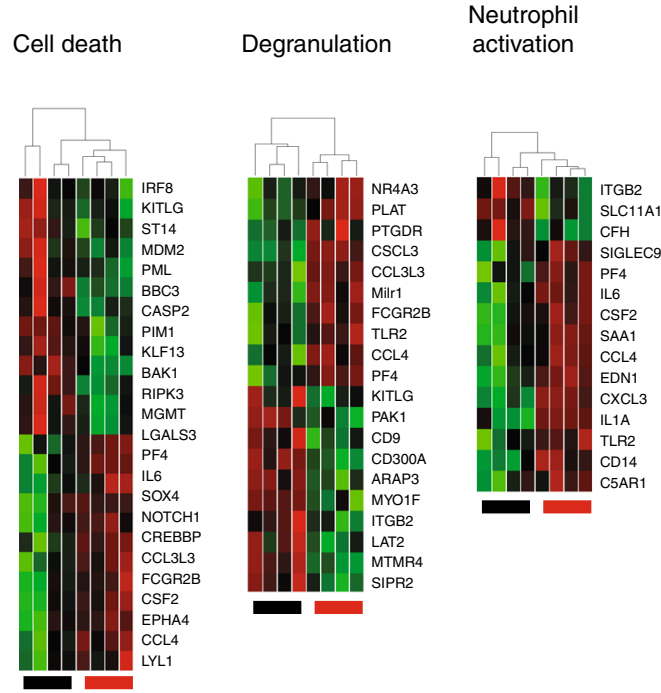

d

\begin{tabular}{|c|c|c|c|c|}
\hline $\begin{array}{c}\text { Upstream } \\
\text { regulator }\end{array}$ & $\begin{array}{c}\text { Molecule } \\
\text { type }\end{array}$ & $p$-value & $\begin{array}{c}\text { Predicted } \\
\text { activation } \\
\text { state }\end{array}$ & $\begin{array}{c}\text { Activation } \\
\text { z-score }\end{array}$ \\
\hline CCL5 & Cytokine & $4.52 \mathrm{E}-07$ & Activated & 2.542 \\
IL1 & Group & $6.16 \mathrm{E}-06$ & Activated & 2.172 \\
TLR2 (family) & $\begin{array}{c}\text { Group } \\
\text { Transmembrane } \\
\text { receptor }\end{array}$ & $1.98 \mathrm{E}-02$ & Activated & 2.012 \\
TLR5 & $\begin{array}{c}\text { Transmembrane } \\
\text { receptor } \\
\text { Transmembrane } \\
\text { receptor }\end{array}$ & $3.08 \mathrm{E}-06$ & Activated & 2.797 \\
NFE2L2 & $\begin{array}{c}\text { Transmembrane } \\
\text { receptor } \\
\text { Transcription } \\
\text { regulator }\end{array}$ & $1.03 \mathrm{E}-03$ & Activated & 2.172 \\
\hline
\end{tabular}

gene sets found in both BH4-deficient and iNOS-deficient macrophages, hinting that NO production by iNOS may also modulate its interaction with other proteins. The endothelin system is a key effector of NOS enzymes in other cell types and also showed alterations in gene expression levels in the absence of $\mathrm{BH} 4$, but expression of both protective (ET-1) and non-protective (ETB) elements of the system were downregulated ${ }^{43}$. So whilst some b
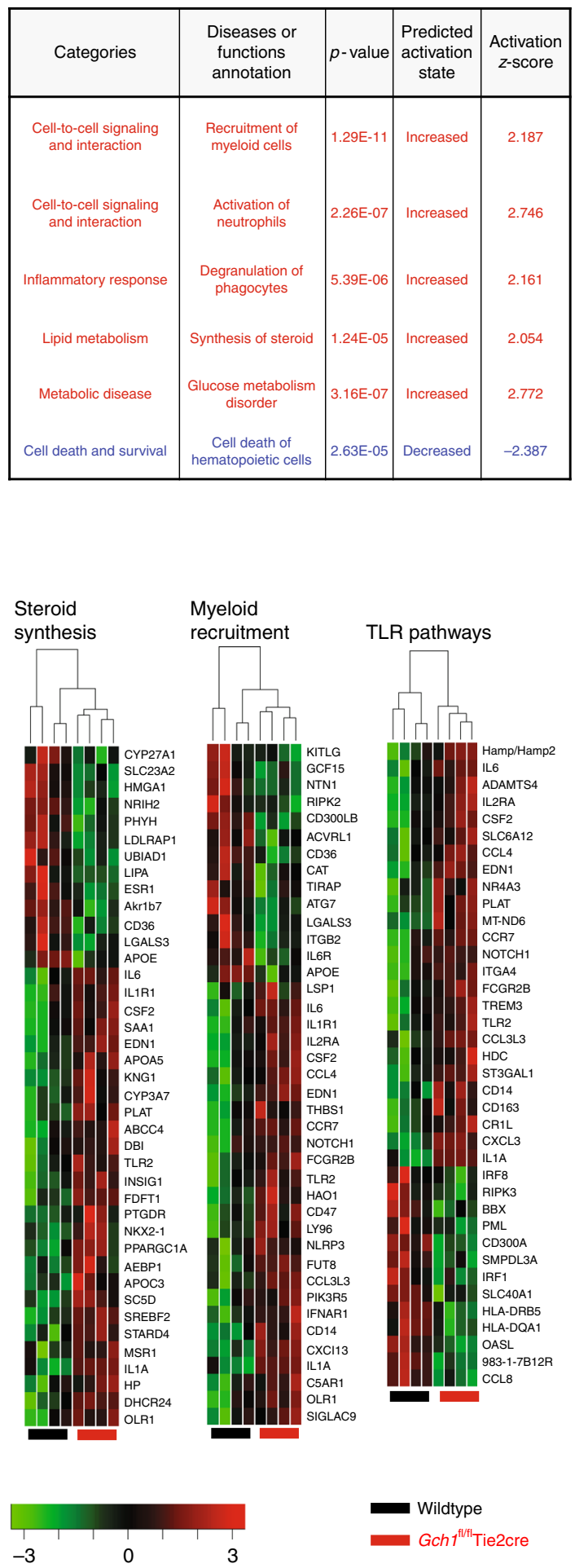

signaling functions attributed to iNOS are linked to nitric oxide, the potential contribution of other signaling pathways is overlooked. The critical difference between our study and previously published analyses of the Nos $2^{-1-}$ mouse is that selective macrophage $\mathrm{BH} 4$ deficiency results in loss of $\mathrm{NO}$ production, rather than loss of all potential iNOS functions, and this results in increased control of mycobacterial infection. 
Fig. 4 Absence of Gch1 alters macrophage gene expression in response to infection. Macrophages were infected with BCG (MOI 1:1)/IFN $(10$ ng/ml) for $24 \mathrm{~h}$ or incubated for $24 \mathrm{~h}$ in the absence of infection ( $n=4$ per genotype). RNA was extracted and a whole mouse genome gene expression array was performed. a Genes were determined as significantly regulated by genotype by comparison to their relevant wildtype control with $p<0.05$ (adjusted for multiple testing). The number of genes significantly regulated by genotype for each condition was calculated. The number of genes shared between genotypes and conditions is shown by Venn Diagram. b Selected relevant gene functional annotations determined with significantly increased or decreased expression in BCG infected wildtype vs Gch ffl/flTie2cre macrophages, as determined using IPA analysis with $Z$ score $>2$ and a $p$-value for overlap $p<0.01$. c Heatmaps for genes within individual gene functional annotations determined in (c, d). d Selected relevant predicted upstream regulators with significantly increased or decreased activation in BCG infected wildtype vs Gch fl/fl/Tie2cre macrophages, as determined using IPA analysis with $Z$ score $>2$ and a $p$-value for overlap $p<0.01$
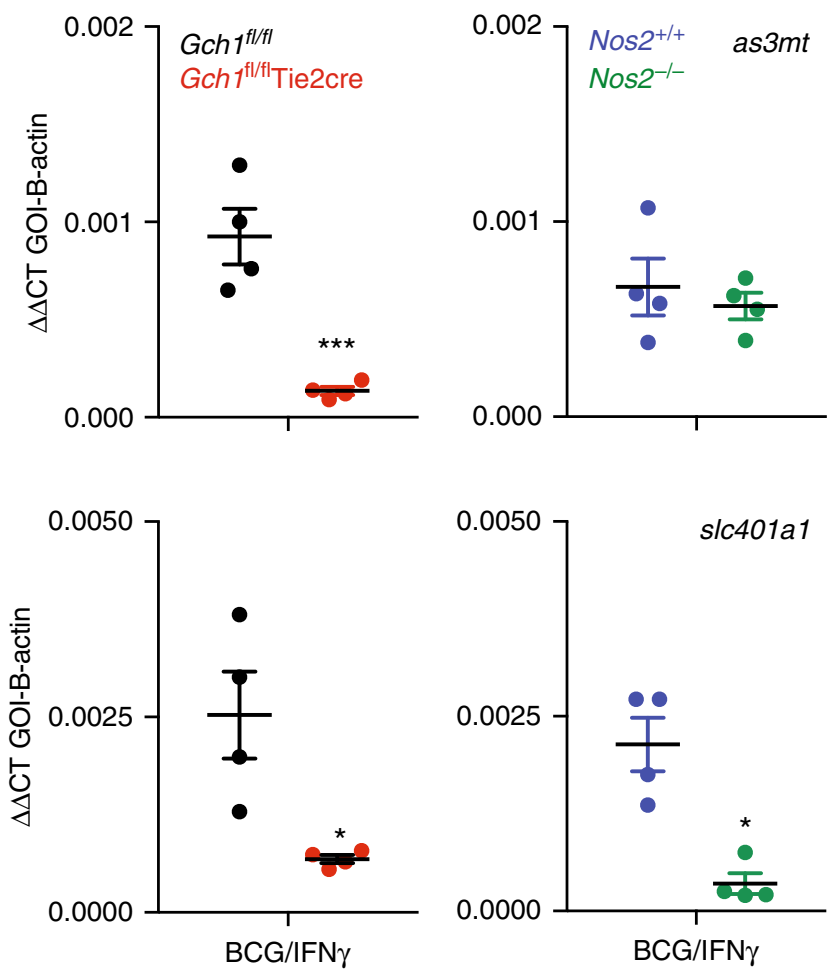

Fig. 5 Divergent gene expression changes in $\mathrm{BH} 4$ and iNOS-deficient macrophages. BMDM were prepared from Gchfl/fl, Gch $f$ l/flTie2cre, Nos2 $2^{+/+}$ and Nos2 $2^{-/}$age-matched animals. Macrophages were infected at a $\mathrm{MOI}$ of 1:1 with BCG in the presence of $10 \mathrm{ng} / \mathrm{ml} \mathrm{IFN \gamma}$, or left uninfected followed by RNA extraction. Gene expression was quantified using TaqMan gene expression assays. GOI Gene of Interest. Data was analysed by two-tailed $T$-test * $p<0.05$, error bars \pm SEM

The loss of macrophage BH4 is sufficient to enhance control of mycobacterial infection by the cell itself. Upon infection with mycobacteria, mycobacterial killing is assumed to occur by the maturation of the uptake phagosome and fusion with the lysosome to form the phagolysosome ${ }^{44}$. While the cytotoxic capacity of the cell was assumed to then reside in the ability to produce $\mathrm{RNI}$ and ROS species, increasingly evidence suggests this is not the case. The role of ROS in direct mycobacterial control is as controversial as the role of nitric oxide. The loss of ROS production in $p 47^{\mathrm{PHOX}-1-}$ mice resulted in only a transient decrease in bacterial control, and human chronic granulomatous disease patients, who lack myeloid cell ROS production, do not show increased susceptibility to $\mathrm{TB}^{45,46}$. An interpretation of our findings is that $\mathrm{Nos}^{-1-}$ cells lack all aspects of iNOS activity (both NO and ROS-mediated), whereas Gch $1^{f l f l}$ Tie2cre cells lack NO production but have preserved ROS production, that may mediate mycobacterial killing. A direct effect of ROS on mycobacterial killing seems less likely, given both the complicated pattern of altered ROS production in the absence of $\mathrm{BH} 4$ and equivocal evidence for mycobacterial killing by ROS. Nevertheless, altered ROS production may exert important effects through altered downstream redox signaling. Increasingly the fate of lysosomal bacteria through the engagement of autophagy and intracellular degradation machinery is highlighted as playing a role in bacterial killing $44,47,48$. Similarly macrophage cell death by apoptosis is associated with decreased viability of mycobacteria ${ }^{49,50}$. The extent to which ROS, nitric oxide and iNOS act as signaling molecules in coordination of these responses may link older and newer findings. The altered lysosomal function and cell survival signals we identified in Gchl deficient macrophages could clearly have implications for mycobacterial control. Additionally, a BH4 specific alteration in metabolic and mitochondrial gene sets in infected macrophages, has similar potential as M.tb causes a profound shift in cellular metabolism towards aerobic glycolysis, which was evident in leukocytes within the lung ${ }^{51}$. Control of mycobacterial infection downstream of IFN $\gamma$ and iNOS was shown to involve activation of aerobic glycolysis, which amplified macrophage activation ${ }^{35}$. Induction of aerobic glycolysis by human macrophages following mycobacterial infection is reported to facilitate control of bacterial replication, with inhibition of this process associated with enhanced mycobacterial survival ${ }^{52}$. As extracellular $\mathrm{H}_{2} \mathrm{O}_{2}$ accumulation has been linked with activity of the mitochondrial electron transport chain the altered $\mathrm{H}_{2} \mathrm{O}_{2}$ accumulation seen following infection in this study may indicate metabolic changes in the absence of macrophage $\mathrm{BH} 4^{53}$.

We have used a genetic approach to identify new mechanisms linking macrophage iNOS and NO to the control of mycobacterial infection in vitro and in vivo, using cell-specific BH4 deficiency, achieved by conditional Gch1 deletion. NOS enzymes can function independently of NO both through protein-protein interactions and in an 'uncoupled' state in the absence of $\mathrm{BH} 4$, whereby L-arginine is not oxidized to $\mathrm{NO}$ and superoxide is produced by the reductase domain of the enzyme $e^{14,15,17}$. Thus, equating NOS activity with nitric oxide production alone potentially overlooks important alternative signaling pathways. Whilst these pathways are dominated by superoxide production from NOS in other BH4-deficient NOS expressing cells, such as endothelial cells ${ }^{28}$, the redox biology of macrophages lacking $\mathrm{BH} 4$ appears more complex. It has previously been reported that $\mathrm{BH} 4$ has important effects on cellular metabolism independent of its effect on NOS biology ${ }^{54}$. Knockdown of Gch1 in the absence of any NOS expression resulted in alterations in bioenergetic metabolism $^{54}$. So whilst the transcriptional effects seen on macrophage metabolism may be important, these may not all be dependent iNOS expression. Tetrahydrobiopterin has other NOSindependent functions as it is also a co-factor for other enzymes such as alkylglycerol monooxygenase (AGMO), which modulates cellular lipid signaling, and catecholamine-related enzymes ${ }^{15,55}$. These NOS-independent roles for $\mathrm{BH} 4$ in macrophage biology are still of particular relevance to humans due to the low levels of BH4 present in human macrophages, such that alterations in cell function relating to both reduced NO-production from iNOS and 
a Effect of Gch1 deletion on BCG/IFN $\gamma$ response

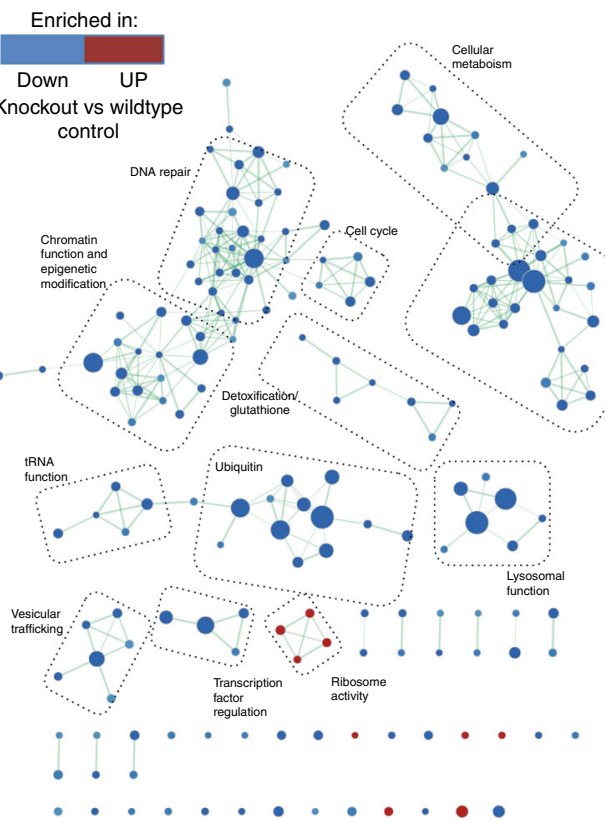

C

Gene-sets unique to the effect of Gch1 deletion

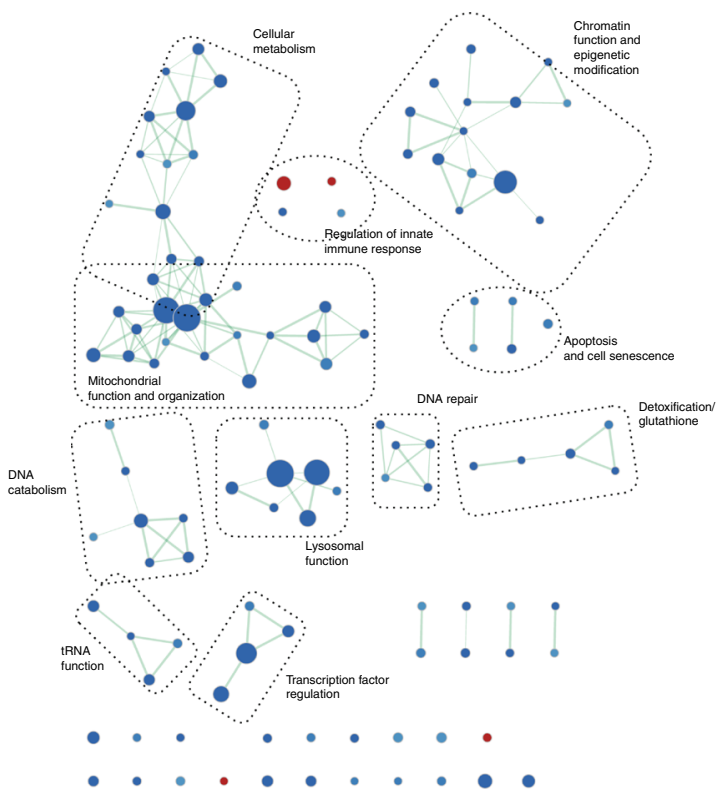

b

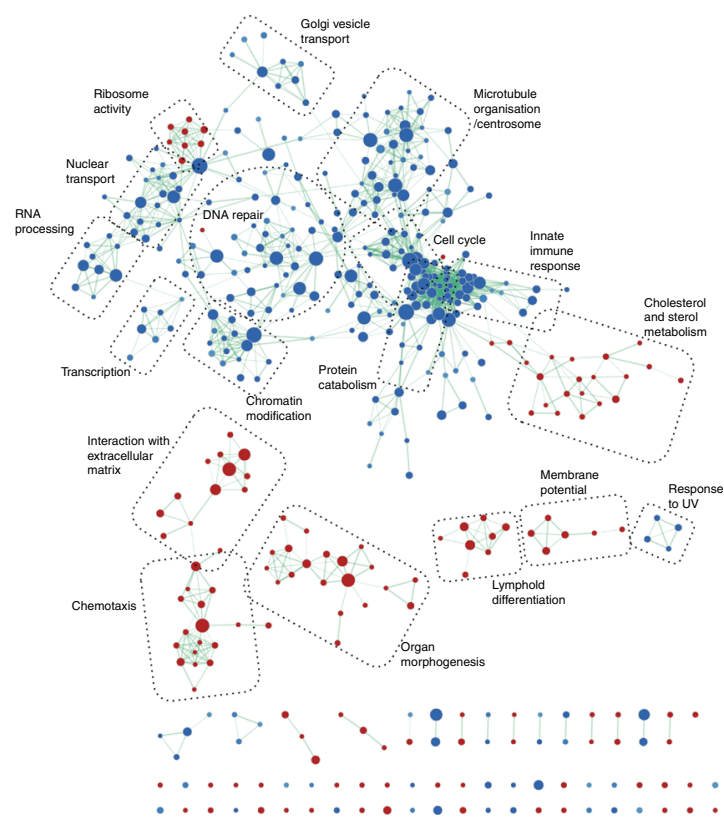

d

\begin{tabular}{|c|c|}
\hline Clusters & Example GO terms \\
\hline Detoxification & $\begin{array}{l}\text { Detoxification, glutathione derivative } \\
\text { metabolic pathways }\end{array}$ \\
\hline DNA repair & $\begin{array}{l}\text { DNA synthesis involved in DNA repair, } \\
\text { mitotic recombination, post replication } \\
\text { repair }\end{array}$ \\
\hline DNA catabolism & $\begin{array}{l}\text { Deoxyribonuclease activity, vacuole } \\
\text { organisation, nuclear transcribed mRNA } \\
\text { catabolic process exonucleolytic }\end{array}$ \\
\hline Lysosomal function & $\begin{array}{l}\text { Lytic vacuole membrane, lysosomal } \\
\text { transport, pH reduction, Iytic vacuole }\end{array}$ \\
\hline tRNA function & $\begin{array}{l}\text { tRNA processing, tRNA methyltransferase } \\
\text { activity }\end{array}$ \\
\hline $\begin{array}{l}\text { Transcription factor } \\
\text { regulation }\end{array}$ & $\begin{array}{l}\text { Regulation of sequence specific DNA } \\
\text { binding transcription factor activity. }\end{array}$ \\
\hline $\begin{array}{l}\text { Chromatin function } \\
\text { and epigenetic } \\
\text { modification }\end{array}$ & $\begin{array}{l}\text { Chromatin silencing, telomere maintenance } \\
\text { by telomerase, protein DNA complex }\end{array}$ \\
\hline $\begin{array}{l}\text { Cellular metabolism } \\
\text { and mitochondrial } \\
\text { function and } \\
\text { organisation }\end{array}$ & $\begin{array}{l}\text { Cofactor metabolic process, oxidative } \\
\text { phosphorylation, mitochondrion } \\
\text { organisation, }\end{array}$ \\
\hline $\begin{array}{l}\text { Apoptosis and cell } \\
\text { senescence }\end{array}$ & $\begin{array}{l}\text { Cellular senescence, intrinsic apoptotic } \\
\text { response to DNA damage }\end{array}$ \\
\hline $\begin{array}{l}\text { Regulations of innate } \\
\text { immune response }\end{array}$ & $\begin{array}{c}\text { Negative regulation of innate immune } \\
\text { response, cytokine activity, acute phae } \\
\text { response }\end{array}$ \\
\hline
\end{tabular}

Fig. 6 GSEA identifies unique gene expression patterns in Gch1 cells. Gene expression data from both Gch1 and Nos2 deficient macrophages were analysed for GO term (biological processes) enrichment by Preranked gene-set enrichment analysis (GSEA) vs their wildtype control. The result was visualized as a network of gene sets (nodes) connected by their similarity (edges), compiled in Cytoscape using the Enrichment Map plug in. Node size represents the gene-set size and edge thickness represents the degree of overlap between neighboring gene sets. The GSEA output files were given analysed with a statistical cutoff set at $p<0.005$ and FDR $q<0.1$. The individual Enrichment Maps were plotted in Cytoscape for infected $\mathbf{a}$ Gch1 and $\mathbf{b}$ Nos2 macrophages and clusters were annotated by hand. To determine if regulated genesets were shared between the Gch1 and Nos 2 knockout macrophages the significantly regulated gene sets were compared and a subset of genesets unique to Gch1 deficient macrophages was created, this subset (c) was plotted using Enrichment Map and the clusters annotated. d A table summarizing the GO-term clusters, with exemplar GO terms

iNOS-independent BH4-dependent changes in macrophage biology have key translational relevance.

We demonstrate an unanticipated aspect of BH4/iNOS biology that mediates enhanced control of mycobacterial infection through mechanisms that are independent of iNOS-mediated NO production. Gch1 and BH4 exert striking effects on the control of mycobacterial infection in macrophages independent of NO-production, in both mice and humans. The BH4-deficient 


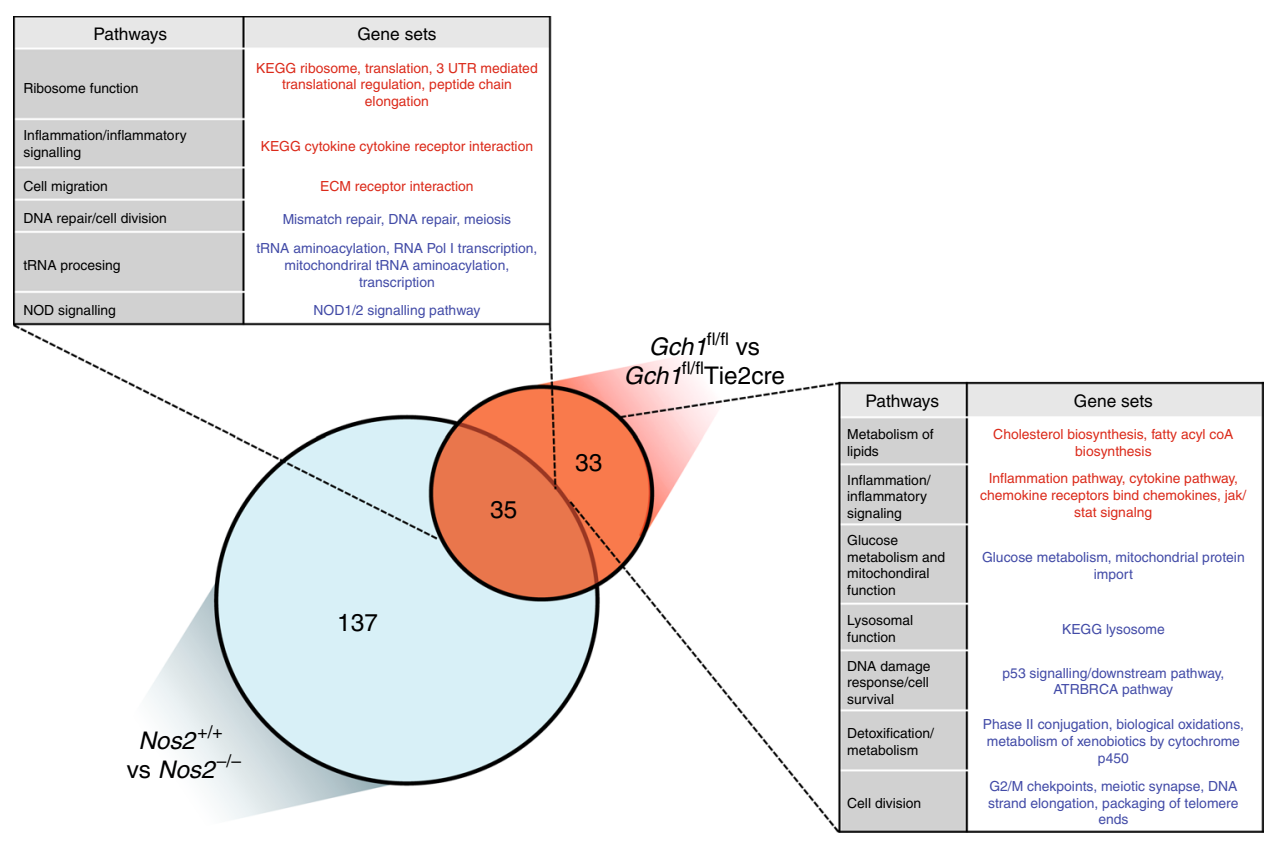

Fig. 7 GSEA using curated pathways identifies shared and unique genesets. Enriched gene sets in BCG infected macrophages, identified by Preranked GSEA (MSigDb, C2 CP annotations set). To determine how far regulated geneset were shared between the Gch1 and Nos2 knockout macrophages or unique to the loss of Gch1 the gene set lists passing $p<0.005$, FDR $q<0.1$ in both analyses were compared and key genesets were are highlighted in the tables
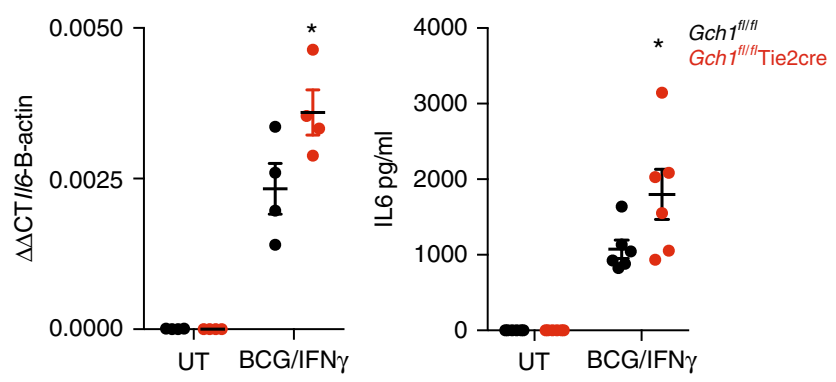

Fig. 8 Gene expression changes result in functional changes is cytokine expression. Macrophages were infected with BCG (MOI 1:1)/IFN $\gamma$ $(10 \mathrm{ng} / \mathrm{ml})$ for $24 \mathrm{~h}$ or incubated for $24 \mathrm{~h}$ in the absence of infection followed by RNA extraction or ELISA analysis of the supernatant. IL-6 gene expression was quantified using TaqMan gene expression assays. Data was analysed by two-tailed $t$-test, ${ }^{\star} p<0.05$, error bars \pm SEM

$G c h 1^{f l / f l}$ Tie2cre mouse may provide a better in vivo model to investigate novel human therapeutics and vaccinations than either wildtype mice, or Nos $2^{-1-}$ mice, due to the strong parallels with human macrophage biology. Exploiting this model could provide novel targets for HDT to control mycobacterial infection that are not possible to study in traditional models.

\section{Methods}

Infant PBMC samples. The correlates of risk samples used in this study formed part of larger cohort from a phase IIb efficacy trial (ClinicalTrials.gov number NCT00953927) ${ }^{33}$. The trial was approved by the University of Cape Town, Faculty of Health Sciences, Human Research Ethics Committee, Oxford University Tropical Research Ethics Committee and the Medicines Control Council of South Africa. Informed consent was obtained from the mothers of all infants. Briefly, 2797 healthy infants (4-6 months) who had received BCG vaccination within 7 days of birth were randomly divided into either receiving MVA85A $(n=1399)$ or Candida as placebo $(n=1398)$. Infants were monitored for up to 37 months. The primary outcome of this study was safety but the efficacy of MVA85A vaccine was also assessed, with primary study endpoint the incidence of tuberculosis. MVA85A did not significantly improve BCG efficacy $(17.3 \%, 95 \%$ CI -31.9 to 48.2$)$. Some of these samples were used in an identification of correlates of risk study $(n=258)^{56}$.
A number of assays (on a priority list) were performed on whole blood and PBMC samples from day of enrolment (D0) and 28 days post vaccination (D28). The MGIA assay was only performed if enough cells were available. For the analysis in this manuscript, PBMC samples $(n=78)$ from D0 were used to avoid confounding effects of MVA85A vaccination.

Animal studies. All animal studies were conducted with ethical approval from the Local Ethical Review Committee at the University of Oxford and in accordance with the UK Home Office regulations (Guidance on the Operation of Animals, Scientific Procedures Act, 1986). Mice were housed in ventilated cages with a 12-h light/dark cycle and controlled temperature $\left(20-22^{\circ} \mathrm{C}\right)$, and fed normal chow and water ad libitum. For in vivo experiments adult female mice $>10$ weeks of age were used for all studies, whole litters were randomly assigned to experimental groups, with heterozygous breeding strategies providing a mix of genotypes within each litter. Experiments using littermate animals were performed blinded to the genotypes of the individual animals. For homozygous strains in vivo experiments were blinded to the genotype by labeling of the cages by a group code, rather than genotype. Mice had received no prior procedures (including acting as breeding stock) prior to use in experiments for this manuscript.

Gch $1^{f l / f l}$ Tie2cre and Gchfl/fl mice. A novel mouse model of BH4 deficiency was created by conditional deletion of Gch1, that encodes GTP cyclohydrolase I the rate-limiting enzyme in $\mathrm{BH} 4$ biosynthesis. Exons 2 and 3 of Gch1, encoding for the active site of GTPCH protein, were flanked by loxP sites in a targeting construct that was used to produce Gch $1^{f l f l}$ mice following homologous recombination in ES cells. Mice have been backcrossed for $>8$ generations to C57BL/6J. These mice were crossed with Tie2cre transgenic mice to produce Gch $1^{f / f l}$ Tie2cre mice where Gchl is deleted in endothelial and bone marrow-derived cells ${ }^{28}$. The Tie2cre transgene is active in the female germline, as such only male Tie2cre heterozygote animals are used to establish breeding pairs to maintain conditional expression. Mice were genotyped using primers targeted against the floxed Gchl allele, and in a separate reaction for the presence of the cre sequence. Experiments were performed using bone marrow isolated from adult (>10-week old) female littermate animals. Animals were genotyped using DNA prepared from ear notches using the following PCR primers:

Cre:

5' GCATAACCAGTGAAACAGCATTGCTG 3'

5' GGACATGTTCAGGGATCGCCAGGCG 3'

Gch1 floxed allele:

5' GTCCTTGGTCTCAGTAAACTTGCCAGG 3'

5' GCCCAGCCAAGGATAGATGCAG 3'

Nos2 ${ }^{-1-}$ mice. Nos $2^{-/-}$mice and C57BL/6J control mice were obtained from Jackson Labs (Bar Harbor, US) and Charles River (UK) $)^{57}$. Experiments were 
a
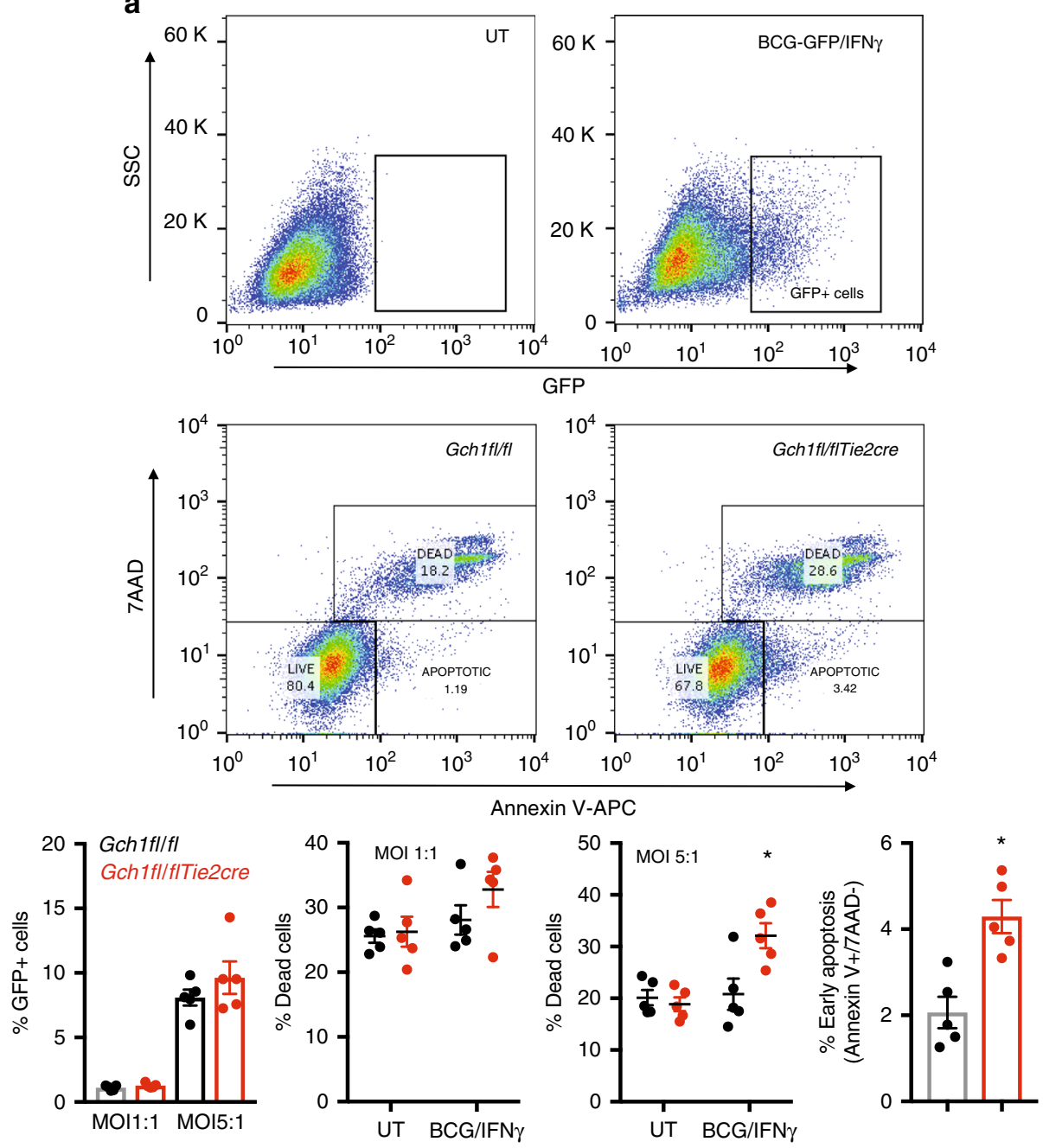

b
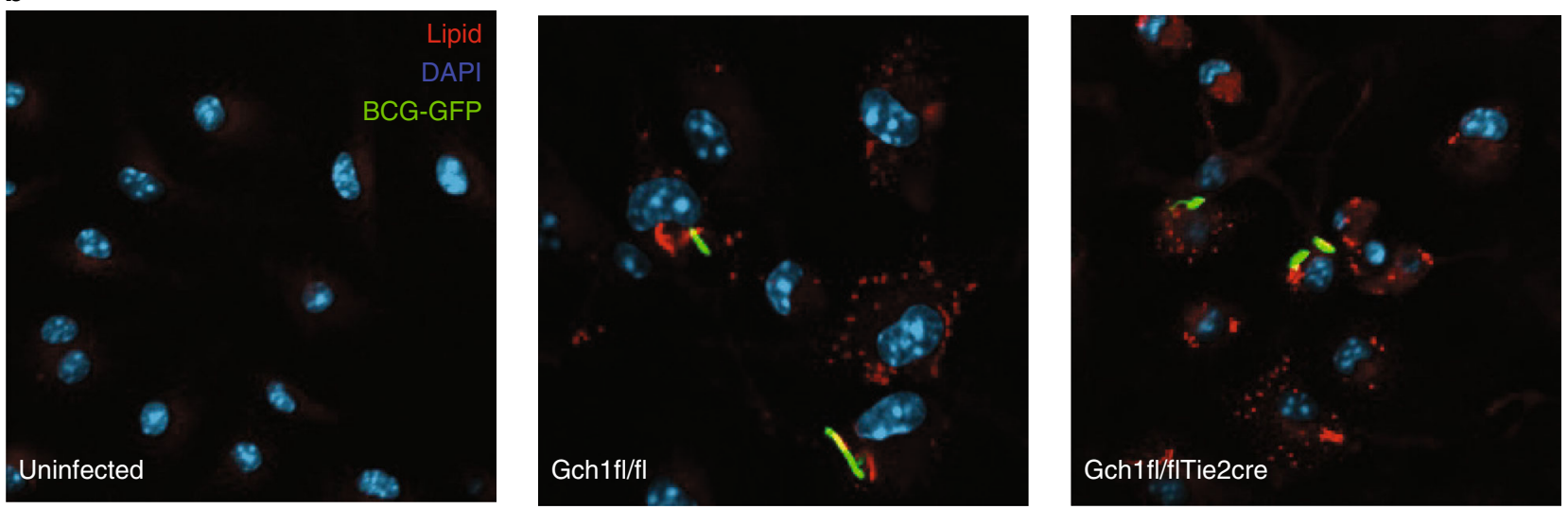

Fig. 9 BH4 deficiency alters infected cell viability. a Gch $7^{\mathrm{fl} / \mathrm{fl}} \mathrm{Tie} 2 \mathrm{cre}$ and $\mathrm{Gch} \mathrm{fl}^{\mathrm{fl} / \mathrm{fl}}$ macrophages were stimulated IFN (10 ng/ml) and BCG (MOI 1:1 or 5:1) or left untreated overnight. At the end of the assay the cells were harvested and incubated in buffer containing 7AAD and Annexin V-APC for 15 min at room temperature. Staining was terminated by addition of buffer and cells were analysed by flow cytometry immediately. Cell death was defined as Annexin $\mathrm{V}^{+} / 7 \mathrm{AAD} \mathrm{D}^{+}$. Infection was confirmed by detection of GFP and early apoptosis (Annexin $\mathrm{V}^{+} / 7 \mathrm{AAD}^{-}$) was assessed in BCG-GFP+ cells infected at MOI 5:1. Data was analysed by 2 -way ANOVA * $p<0.05$ with Bonferroni post-test or two-tailed $t$-test ( $n=5 /$ genotype). b Gch fl/flTie2 $\mathrm{cre}$ and Gch $7 \mathrm{fl} / \mathrm{fl}$ macrophages were stimulated with IFN $\gamma(10 \mathrm{ng} / \mathrm{ml})$ and BCG (MOI 1:1) or left untreated overnight in the presence of $2 \%$ serum as a lipid source. At the end of the assay the cells were fixed and neutral lipids were stained using LipidTox dye and nuclei stained with DAPI. Images are representative of 4 individual macrophage cultures. Error bars \pm SEM 

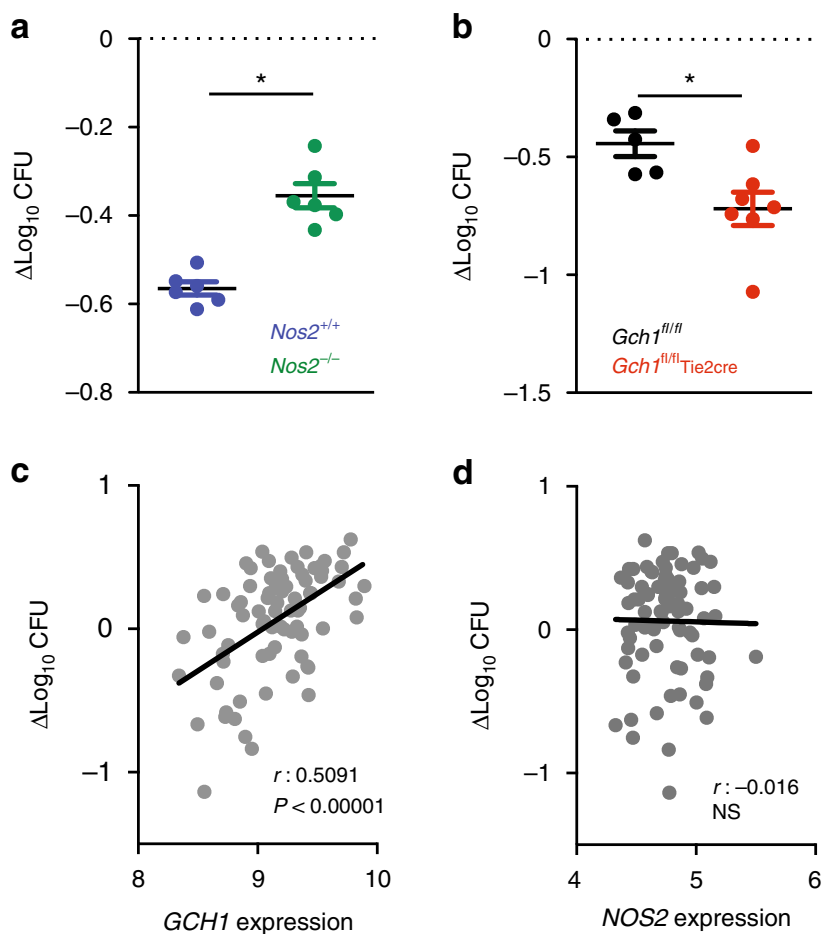

Fig. 10 Gch1 expression inversely correlates with control of BCG infection in vitro. a, b A mycobacterial growth inhibition assay was performed using Nos2 $2^{-/-}$(a) and $\mathrm{Gch}^{-/}$(b) BMDM cells. Macrophages were infected with 50:1 MOI BCG in the presence of $10 \mathrm{ng} / \mathrm{ml} \mathrm{IFN} \gamma$ and the live BCG remaining after $96 \mathrm{~h}$ was enumerated by the BACTEC Mycobacterial Growth Indicator Tube (MGIT) system (MGIA), $n=6$ per genotype Nos2 $2^{+/+}$and Nos2 $2^{-1-}$,

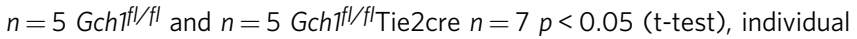
points with SEM shown. $\Delta \log _{10} \mathrm{CFU}=\log _{10} \mathrm{CFU}$ sample $-\log _{10} \mathrm{CFU}$ positive control. Error bars are \pm SEM. c, d Pearson correlation between GCH1 expression and in vitro BCG growth in PBMCs of 78 BCG vaccinated South African infants. Points represent the mean of duplicate cultures. $\Delta \log _{10}$ CFU $(M . B C G)=\log _{10}$ CFU of sample $-\log _{10}$ CFU of control

performed using bone marrow isolated from adult ( $>10$-week old) female littermate animals.

Isolation of murine bone marrow-derived macrophages. Bone marrow was obtained by flushing the femur and tibia of adult female mice with PBS. A single cell suspension was prepared by passing the bone marrow through a $70 \mu \mathrm{m}$ cell strainer. Cells were then cultured in $10 \mathrm{~cm}$ non-tissue culture treated dishes for 7 days in DMEM:F12 (Invitrogen) supplemented with $100 \mathrm{U} / \mathrm{ml}$ penicillin and $100 \mathrm{ng} / \mathrm{ml}$ streptomycin (Sigma), 10\% (v/v) fetal bovine serum (PAA Laboratories), $5 \mathrm{mM}$ L-glutamine (Sigma) and 10-15\% (v/v) L929 (ATCC NCTC clone 929) conditioned medium at $37^{\circ} \mathrm{C}$ and $5 \% \mathrm{CO}_{2}$. The protocol for macrophage culture was validated by flow cytometry analysis of the differentiated cells using f $4 / 80$ and $\mathrm{CD} 11 \mathrm{~b}$ as macrophage cell markers.

Blood, bone marrow and spleen leukocytes. Single cell suspensions of splenocytes and bone marrow cells were obtained using standard protocols ${ }^{58}$. Blood samples were taken directly into an EDTA coated tube (Teklab). All cell populations were stained with monoclonal antibodies directed against CD11b PerCPCy5.5 (550993), CD4 AF647 (557681), CD8 FITC(553030), CD3 Pacific Blue (558214), B220 PE(553089), Ly-6G FITC(561105) (all BD Biosciences, 1/200) and the $7 / 4$ antigen PE (MCA771PE, AbD Serotech, 1/50). The total cell population was gated by forward scatter and side scatter then interrogated for expression of the relevant cells markers compared to isotype controls (T-cells-CD3 ${ }^{+}$, B-Cells$\mathrm{B} 220^{+}$, Neutrophils-7/4 $\mathrm{HI}, \mathrm{Ly}-6 \mathrm{G}^{+}$or Monocytes $\left.-7 / 4^{\mathrm{HI}}, \mathrm{Ly}-6 \mathrm{G}^{-}\right)$. Cells were enumerated by an absolute count as described below.

Zymosan-induced peritonitis. Mice were injected with $1 \mathrm{mg}$ zymosan (Sigma) in $500 \mu \mathrm{l}$ PBS using an insulin syringe. After $6 \mathrm{~h}$ mice were lavaged post-mortem with ice-cold PBS/EDTA $(5 \mathrm{mM})$, lavage fluid was injected using a $25 \mathrm{G}$ needle, the cavity mixed by agitation, and then lavage fluid was withdrawn using a $21 \mathrm{G}$ needle. Cell recruitment was assessed by flow cytometry using 7/4-PE and Ly-6G-FITC using an absolute cell count method as below. Total cell recruitment was calculated as the total cells that would be recovered in the $5 \mathrm{ml}$ lavage volume.

Bacterial culture. BCG Pasteur (ATCC 35734) was grown in-house in Middlebrook 7H9 Broth (BD, UK) supplemented with Middlebrook ADC $+0.05 \%$ Tween 80. Erdman K01 (TMC107) was provided by BEI Resources (NR-15404, BEI Resources; Manassas, USA). BCG-GFP was grown under the same conditions but in the presence of Hygromycin B $(50 \mu \mathrm{g} / \mathrm{ml})$ to select GFP expressing bacteria.

Flow cytometry. All flow cytometry was performed using a DAKO CyAn cytometer and Summit software (both Beckton Coulter). Data was analysed using Flow Jo software (TreeStar Inc). Where cells were enumerated using an absolute count protocol with cells quantified by ratio to a known number of fluorescent beads spiked into the sample prior to analysis.

Stimulation of bone marrow-derived macrophages. Following differentiation cells were harvested and plated into 6 or 12 well plates in serum-free media (Optimem supplemented and $0.2 \%(\mathrm{w} / \mathrm{v})$ low-endotoxin bovine serum albumin (Sigma)). Cells were stimulated with BCG at a MOI of 1:1 in the presence of $10 \mathrm{ng} / \mathrm{ml}$ murine IFN $\gamma$ (Peprotech EC) for $24 \mathrm{~h}$, parallel wells were left unstimulated. After $24 \mathrm{~h}$ cell pellets and cell culture supernatants were collected, or the cells subjected to biochemical analysis.

Genomic DNA production and excision PCR. Genomic DNA for detection of the excised allele was produced using the Qiamp kit (Qiagen). The floxed and excised allele were detected using the following primers:

5'GTCCTTGGTCTCAGTAAACTTGCCAGG3'

5'GCCCAGCCAAGGATAGATGCAG3'

5'GCTCATCCCCCACACTTGTCTT3'

The floxed allele yields a $1030 \mathrm{bp}$ product and the excised allele a product of $1300 \mathrm{bp}$.

Determination of tetrahydrobiopterin levels. BH4 and oxidized biopterins (BH2 and biopterin) were determined by high-performance liquid chromatography (HPLC) followed by electrochemical and fluorescent detection, respectively ${ }^{59}$. Cell pellets were freeze-thawed in ice-cold resuspension buffer $(50 \mathrm{mM}$ phosphatebuffered saline, $1 \mathrm{mM}$ dithioerythritol, $1 \mathrm{mM}$ EDTA, $\mathrm{pH}$ 7.4). After centrifugation at $13,200 \mathrm{rpm}$ for $10 \mathrm{~min}$ at $4{ }^{\circ} \mathrm{C}$, supernatant was removed and ice-cold acid precipitation buffer (1 M phosphoric acid, $2 \mathrm{M}$ trichloroacetic acid, $1 \mathrm{mM}$ dithioerythritol) was added. Following centrifugation at $13,200 \mathrm{rpm}$ for $10 \mathrm{~min}$ at $4^{\circ} \mathrm{C}$, the supernatant was removed and injected onto the HPLC system. Quantification of $\mathrm{BH} 4$ and oxidized biopterins was obtained by comparison with external standards and normalized to protein concentration, determined by the BCA protein assay. Total biopterins ( $\mathrm{BH} 4+\mathrm{BH} 2+$ biopterin) were quantified where significant BH4 oxidation might be expected to occur in extracellular fluids/peritoneal lavage or unperfused whole tissue homogenates subjected to harsh homogenization for CFU quantification.

Nitrite and NOx determination. Nitrite accumulation was measured in samples of cell culture medium using the Griess Assay with colorimetric detection in 96-well plates. Cell culture supernatants were mixed 1:1 with the Griess reagent (Sigma) and quantified by comparison to a sodium nitrite (Sigma) standard curve produced in tissue culture media. Measurement of NOx in lavage fluid was made using a Nitrite/Nitrate kit (Cayman Chemicals, US). Analysis of NOx in lung homogenates was performed using a CLD88 NO analyzer (Ecophysics).

Electron paramagnetic resonance. Cultured macrophages were incubated with colloid iron (II) diethyldithiocarbamate $\left[\mathrm{Fe}(\mathrm{DETC})_{2}\right](285 \mu \mathrm{mol} / \mathrm{L})$ at $37^{\circ} \mathrm{C}$ for $90 \mathrm{~min}$. After incubation, cells were harvested and snap-frozen in a column of Krebs-HEPES buffer in liquid nitrogen, and EPR spectra were obtained using an X-band EPR spectrometer (Miniscope MS 200; Magnettech). Signals were quantified by measuring the total amplitude, after correction of baseline, and after subtracting background signals from incubation with colloid $\mathrm{Fe}(\mathrm{DETC})_{2}$ alone.

Dihydroethidium HPLC. Cultured macrophages were incubated with $25 \mu \mathrm{M}$ DHE (Invitrogen) for $30 \mathrm{~min}$ before being harvested for separation of DHE using gradient HPLC system (Jasco, UK) with an ODS3 reverse phase column $(250 \mathrm{~mm}$, $4.5 \mathrm{~mm}$, Hichrom, UK) and quantified using a fluorescence detector set at $510 \mathrm{~nm}$ (excitation) and $595 \mathrm{~nm}$ (emission). Samples for injection were prepared by lysing the cell pellets in ice-cold methanol and removing protein by acid precipitation with $0.1 \mathrm{M} \mathrm{HCl}$.

BCG intranasal infection. For intranasal (i.n.) infection, animals were sedated using IsoFlo (Oxford University Veterinary Services; UK) and inoculated with 
$5 \times 10^{6} \mathrm{CFU}$ BCG through the nostrils $(25 \mu \mathrm{l} /$ nostril). Four weeks after infection, mice were sacrificed and lung and spleen were aseptically removed.

M.tb aerosol challenge. Mice were challenged using a Biaera AeroMP-controlled nebuliser (Biera Technologies; Hagerstown, USA) contained in a Category Level 3 TCOL isolator. Animals were loaded in nose-only restrainers and exposed to aerosolised Erdman K01 (TMC107) (BEI Resources; Manassas, USA), prepared at a $5 \times 10^{6} \mathrm{cfu} / \mathrm{ml}$ in the nebuliser. The program was run for $10 \mathrm{~min}$ plus a $5 \mathrm{~min}$ purge with the airflow set to $12 \mathrm{~L} / \mathrm{min}$ at a pressure of $20 \mathrm{psig}$. The target dose of 100-200 $\mathrm{CFU} / \mathrm{animal}$ was confirmed by sacrificing two infected animals from each run $24 \mathrm{~h}$ post-challenge. For M.tb. challenge studies 8 mice per group were used. Experiments were designed to have $75 \%$ power to detect differences between groups, based on a $p$ value of 0.05 .

Mycobacterial growth inhibition assay. BMDM from all four genotypes were prepared as above. Cells were treated with IFN $\gamma(10 \mathrm{ng} / \mathrm{ml})$ and BCG-Pasteur with a MOI of 50:1. The tubes were incubated at $37^{\circ} \mathrm{C}$ on a $360^{\circ}$ rotator for $96 \mathrm{~h}$. In parallel, a positive control was set up, by adding the same inoculum of BCG, but no cells, in a BACTEC MGIT tube (BD) supplemented with OADC/PANTA supplement (BD). After incubation, sample tubes were spun at 12,000 rpm for $10 \mathrm{~min}$, and cells were lysed with $500 \mu \mathrm{l}$ of water (Sigma) for $10 \mathrm{~min}$, vortexing twice in between. The resulting solution was transferred into a supplemented MGIT and incubated until positive in a BACTEC MGIT 960 machine. Data are expressed as $\Delta \log _{10}$ CFU which is $\log _{10}$ CFU of a sample subtracted by $\log _{10}$ CFU of positive control.

Quantification of CFU. Lungs and spleens of infected animals were harvested after challenge. Organs were homogenised in re-inforced tubes with ceramic beads containing $1 \mathrm{ml}$ PBS using Precellys 24 (Stretton Scientific, UK). Homogenised organs were diluted in PBS and dilutions were plated in Middlebrook 7H10 plates (BD), containing OADC (BD Diagnostic Systems). Plates were incubated at $37^{\circ} \mathrm{C}$ and counted 3 weeks later.

Histology and immunohistochemistry. For TB infected lungs, histology mice were sacrificed by cervical dislocation, a slit was created in the trachea and one lung lobe placed in PBS for confirmation of CFU. A tube was inserted in the trachea and lungs were inflated with $1 \mathrm{ml}$ of $10 \%$ Neutral Buffered Formalin (NBF; Sigma Aldrich, UK). The lungs were removed and placed in $10 \mathrm{ml}$ of NBF overnight at $4{ }^{\circ} \mathrm{C}$ and then transferred into $70 \%$ ethanol and stored at $4{ }^{\circ} \mathrm{C}$

Paraffin-embedded tissue samples were sectioned $(7 \mu \mathrm{m})$ and stained with hematoxylin and eosin (Sigma) and Acid Fast stain (BD Biosciences). Immunohistochemical staining was performed using anti-iNOS antibody (sc-651, Santa Cruz, 1/500) and anti-Galectin 3 (AF1197, R\&D Systems, 1/500). This was followed by a secondary biotinylated antibody (Vector Laboratories, UK) then avidin-biotin-AP complex and visualized with Vector Red alkaline phosphatase substrate (Vector Laboratories, UK)

Western blot analysis. Western blot analysis was performed using antibodies against murine GTPCH (a gift from S. Gross, Cornell University New York, 1/500), iNOS (610329 BD Pharmigen, 1/1000) and $\beta$-Tubulin (ab6046, Abcam, 1/50,000), using standard protocols.

Quantitative real-time RT-PCR. RNA was prepared using the RNeasy kit (Qiagen) and was reverse transcribed using Superscript II (Life Technologies). $50 \mathrm{ng}$ RNA equivalent cDNA was used to perform real-time PCR using pre-designed Taq-Man gene expression assays (Gch1: Mm01322973 m1, Nos2:

Mm00440502_m1, TNFa: Mm00443258_m1, Life Technologies) using a BioRad CFX1000. Gene expression data was normalized to $\beta$-actin expression (4352341E, Life Technologies) using $2^{- \text {(B-actinCT-Gene of interestCT) }}$

IL-6 ELISA. Cell supernatants were harvested and cell debris removed by pelleting at $13,000 \mathrm{rpm}$ in a microfuge. Appropriate dilutions of the supernatant media were performed and samples were analysed using an ELISAMAX Mouse IL-6 ELISA according to the manufacturer's instructions (Biolegend, UK).

Oxidative burst. Cells were infected with BCG-GFP/IFN $\gamma$ for $2 \mathrm{~h}$ in antibiotic-free media supplemented with $2 \%$ FCS (to increase opsonisation) and then stained with $2.5 \mu \mathrm{M}$ CellROX spiked into the media. After 30 min cells were rapidly detached in PBS/EDTA by scraping and cell fluorescence was analysed by flow cytometry.

Hydrogen peroxide measurements. Cell free supernatants were subjected to amperometric measurement of hydrogen peroxide using a Hydrogen Peroxide Microsensor electrode and Free Radical Analyser system (WPI, UK).

Apoptosis. Cells were infected with BCG-GFP/IFN $\gamma$ for $2 \mathrm{~h}$ in antibiotic-free media supplemented with $2 \%$ FCS (to increase opsonisation) and then harvested using ice-cold PBS/EDTA $5 \mathrm{mM}$. Cells were washed and stained with an 7AAD and Annexin V-APC apoptosis kit according to the manufacturer's instructions (Biolegend, UK).

Lipid droplet formation. Cells were infected with BCG-GFP/IFN $\gamma$ overnight in antibiotic free media supplemented with $2 \%$ FCS (to increase opsonisation and as a lipid source). Cells were washed and fixed with $4 \%$ PFA for $15 \mathrm{~min}$. Cells were then washed and incubated in PBS containing LipidTOX Red Neutral Lipid Stain (Invitrogen, UK) at 1:500 dilution for a minimum of $30 \mathrm{~min}$. Nuclei were costained using $1 \mu \mathrm{g} / \mathrm{ml}$ DAPI (Merck, UK) and cells were imaged in glass cover-slip bottomed dishes without mounting using an inverted confocal microscope. (LSM510, Carl Zeiss, Germany).

Human PBMC MGIA. For the mycobacterial growth inhibition assay, $1 \times 10^{6}$ PBMC were inoculated with $\sim 600 \mathrm{CFU}$ of BCG Pasteur in a volume of $600 \mu \mathrm{l}$ cell culture medium (RPMI-1640 containing 10\% pooled human serum, $2 \mathrm{mM} \mathrm{l-}$ glutamine and $25 \mathrm{mM}$ HEPES). Samples were cultured in duplicate in $2 \mathrm{ml} \mathrm{screw-}$ cap microtubes and incubated at $37^{\circ} \mathrm{C}$ for $96 \mathrm{~h}$ with $360^{\circ}$ rotation. Cells were then lysed using sterile water, and the lysate transferred to a BACTEC MGIT tube supplemented with PANTA/enrichment supplement (Becton Dickinson, UK). The tubes were placed in a BACTEC MGIT 960 and incubated until positivity. The time to positivity (TTP) was then converted to $\log _{10} \mathrm{CFU}$ using stock standard curves of TTP against inoculum volume and CFU. For each batch of samples, a direct-toMGIT control was run in duplicate to correct for day-to-day variability in inoculum using the following calculation: $\log _{10} \mathrm{CFU}$ of sample $-\log _{10} \mathrm{CFU}$ of growth control.

Human gene expression microarrays. Microarrays on the infant PBMC samples were performed as described previously ${ }^{60}$. Briefly, RNA was extracted from thawed PBMCs using the RNeasy kit (Qiagen) according to manufacturer's instructions. mRNA was amplified using the Illumina Total prep kit (Ambion) according to manufacturer's instructions. RNA was labelled and hybridised to Illumina Human HT-12 v4 beadchips which were scanned on an Illumina iScan machine and data were extracted using the GenomeStudio software. Raw, probe level summary values were imported into $\mathrm{R}$ using beadarray ${ }^{61}$. Probes were background corrected using negative control probes followed by quantile normalization using the neqc command $^{62}$.

Mouse gene expression microarray analysis. Total RNA was extracted from each sample using a mirVANA kit (Ambion). RNA was quantified using a nanodrop spectrophotomoter and adjusted to $100 \mathrm{ng} / \mathrm{ul}$. RNA quality control was performed using an Aglient Tape Station to confirm RNA integrity and concentration. RNA was reverse transcribed, converted into labelled cRNA, frag mented and used for hybridization. A genome wide analysis of gene expression was performed by hybridising the total RNA samples using the GeneChip WT PLUS assay for GeneTitan on the Mouse Gene 1.1 ST 16-Array plate system. The Affymetrix Genechip Command Console software 4.0 for GeneTitan was used to carry out QC checks of the procedure and produce reports.

Data underwent variance stabilization and normalization (VSN) algorithm using Limma ${ }^{63}$. Normalized data were imported to Bioconductor (R version 2.14) (Biobase) and comparison by genotype was performed for each condition (infected and control) to identify significantly differentially expressed genes passing a false discovery rate of $<0.05^{64}$. The effect of infection was confirmed by comparison within the wildtype animals for infected vs control. The lists of differentially expressed genes were then subject to IPA pathway analysis (Ingenuity Systems, http://www.ingenuity.com). Due to the large number of significantly regulated genes for the effect of infection the top 2538 most significant genes were used for IPA analysis $\left(p<1 \times 10^{-7}\right)$. For analysis of the effect of loss of $g c h 1$ or nos 2 those genes passing adj $p<0.05$ were used. Analysis of gene function or upstream regulators was performed and categories with $Z$ score $>2 /-2$ (allowing directionality to be determined) and an overlap $p$ value $<0.05$ were considered significant.

For Gene Set Enrichment Analysis (GSEA) preranked lists for the effect of genotype within infected samples were created. GenePattern v3.9.10 (Broad Institute) was used and lists were subjected to analysis the GSEAPreranked module using the GO biological processes gene sets (C5 BP) and Curated Pathways (C2 CP) from the Molecular Signature Database v6.0 (MSigDB) ${ }^{65-67}$. Gene Sets with a FDR $q<0.1$ and $p$ value $p<0.005$ were considered significant in this analysis and used in further analyses. Network analysis of significantly regulated gene sets $(p<$ 0.005 and FDR $q<0.1$ ) was performed using Cytoscape v3.5.1 and the Enrichment map plugin ${ }^{30,68,69}$, the Enrichment Map default overlap coefficient of 0.5 was used, as described in ref. ${ }^{30}$. To create a list of gene sets unique to the loss of Gchl any genes passing $p<0.005$ and FDR $q<0.1$ present in both Gch1 and Nos2 deficient macrophage analyses were removed from the Gchl list and the remaining gene sets replotted in Cytoscape.

Quantification and statistical analysis. Statistical significances are indicated in figures $\left({ }^{*} p<0.05\right)$ and mentioned in the figure legends. Data were statistically significant when $p<0.05$. Statistical tests used are indicated in the figure legends 
and were calculated with Graph Pad Prism 6. Venn Diagrams were produced using BioVenn.nl ${ }^{70}$. Indicated $n$-values in figure legends represent individual biological replicates, i.e. individual mice or cell culture derived from different individuals. Bar chart and individual point data are expressed as mean \pm SEM, unless stated in the figure legends. Comparisons between WT and $G C H 1^{f l / f} / \mathrm{Tie} 2 \mathrm{cre}$ were made by twoway ANOVA with Bonferroni post-tests between individual groups or two-tailed $t$ test (2 sample groups). Where two sample groups were compared by unpaired $t$ test an $F$ test was also performed to compare variance, where this indicated that variances were significantly different Welch's corrected $t$-test was used. In this study Welch's correction was applied to gene expression data. Time course studies were compared by ANOVA for repeated measurements. CFU studies were analysed by Mann-Whitney test, with statistical outliers identified using Grubb's test and removed from the dataset. In this case a single value was removed from the spleen CFU values in Fig. 2. Correlation data was analysed using Pearson's correlation coefficient within Prism 6, both $r^{2}$ and $p$ value are reported.

\section{Data availability}

For the human studies, raw and normalized expression data have been deposited at Gene Expression Omnibus under the accession number GSE98550. For the mouse studies, raw and normalized expression data have been deposited at GEO under the accession number GSE107543. Source data for western blot and DNA gels and flow cytometry gating in Figures 1 and 2 is available in the supplementary methods file. Full lists of genes and genesets passing significance thresholds are provided as supplementary datasets. A reporting summary for this article is available as a Supplementary Information file.

Received: 8 November 2017 Accepted: 21 November 2018 Published online: 20 December 2018

\section{References}

1. World Health Organisation. Global Tuberculosis Report 2016 (2016).

2. Qu, H. Q., Fisher-Hoch, S. P. \& McCormick, J. B. Molecular immunity to mycobacteria: knowledge from the mutation and phenotype spectrum analysis of Mendelian susceptibility to mycobacterial diseases. Int. J. Infect. Dis. 15, e305-313 (2011).

3. Gomez, L. M. et al. A polymorphism in the inducible nitric oxide synthase gene is associated with tuberculosis. Tuberculosis 87, 288-294 (2007).

4. Garcia, I. et al. Lethal Mycobacterium bovis Bacillus Calmette Guerin infection in nitric oxide synthase 2-deficient mice: cell-mediated immunity requires nitric oxide synthase 2. Lab. Invest. 80, 1385-1397 (2000).

5. MacMicking, J. D. et al. Identification of nitric oxide synthase as a protective locus against tuberculosis. Proc. Natl. Acad. Sci. U.S.A. 94, 5243-5248 (1997).

6. Scanga, C. A. et al. The inducible nitric oxide synthase locus confers protection against aerogenic challenge of both clinical and laboratory strains of Mycobacterium tuberculosis in mice. Infect. Immun. 69, 7711-7717 (2001).

7. Cooper, A. M., Pearl, J. E., Brooks, J. V., Ehlers, S. \& Orme, I. M. Expression of the nitric oxide synthase 2 gene is not essential for early control of Mycobacterium tuberculosis in the murine lung. Infect. Immun. 68, 6879-6882 (2000).

8. Denis, M. Interferon-gamma-treated murine macrophages inhibit growth of tubercle bacilli via the generation of reactive nitrogen intermediates. Cell. Immunol. 132, 150-157 (1991).

9. Chan, J., Xing, Y., Magliozzo, R. S. \& Bloom, B. R. Killing of virulent Mycobacterium tuberculosis by reactive nitrogen intermediates produced by activated murine macrophages. J. Exp. Med. 175, 1111-1122 (1992).

10. Long, R., Light, B. \& Talbot, J. A. Mycobacteriocidal action of exogenous nitric oxide. Antimicrob. Agents Chemother. 43, 403-405 (1999).

11. Bogdan, C. Nitric oxide synthase in innate and adaptive immunity: an update. Trends Immunol. 36, 161-178 (2015)

12. Tzeng, E., Billiar, T. R., Robbins, P. D., Loftus, M. \& Stuehr, D. J. Expression of human inducible nitric oxide synthase in a tetrahydrobiopterin (H4B)deficient cell line: H4B promotes assembly of enzyme subunits into an active dimer. Proc. Natl. Acad. Sci. U.S.A. 92, 11771-11775 (1995).

13. MacMicking, J., Xie, Q. W. \& Nathan, C. Nitric oxide and macrophage function. Annu. Rev. Immunol. 15, 323-350 (1997).

14. Vasquez-Vivar, J. et al. Superoxide generation by endothelial nitric oxide synthase: the influence of cofactors. Proc. Natl. Acad. Sci. U.S.A. 95, 9220-9225 (1998).

15. Bendall, J. K., Douglas, G., McNeill, E., Channon, K. M. \& Crabtree, M. J. Tetrahydrobiopterin in cardiovascular health and disease. Antioxid. Redox Signal. 20, 3040-3077 (2014).

16. McNeill, E. \& Channon, K. M. The role of tetrahydrobiopterin in inflammation and cardiovascular disease. Thromb. Haemost. 108, 832-839 (2012).

17. Foster, M. W. et al. Proteomic analysis of the NOS2 interactome in human airway epithelial cells. Nitric Oxide 34, 37-46 (2013).
18. Schneemann, M. \& Schoeden, G. Macrophage biology and immunology: man is not a mouse (vol 81, pg 579, 2007). J. Leukoc. Biol. 81, 1334-1334 (2007).

19. Fang, F. C. \& Nathan, C. F. Man is not a mouse: reply. J. Leukoc. Biol. 81, 580 (2007).

20. Schneemann, M. \& Schoedon, G. Species differences in macrophage NO production are important. Nat. Immunol. 3, 102 (2002).

21. Nicholson, S. et al. Inducible nitric oxide synthase in pulmonary alveolar macrophages from patients with tuberculosis. J. Exp. Med. 183, 2293-2302 (1996).

22. Choi, H. S., Rai, P. R., Chu, H. W., Cool, C. \& Chan, E. D. Analysis of nitric oxide synthase and nitrotyrosine expression in human pulmonary tuberculosis. Am. J. Respir. Crit. Care Med. 166, 178-186 (2002).

23. Leitner, K. L. et al. Low tetrahydrobiopterin biosynthetic capacity of human monocytes is caused by exon skipping in 6-pyruvoyl tetrahydropterin synthase. Biochem. J. 373, 681-688 (2003).

24. Tatham, A. L. et al. GTP cyclohydrolase I expression, protein, and activity determine intracellular tetrahydrobiopterin levels, independent of GTP cyclohydrolase feedback regulatory protein expression. J. Biol. Chem. 284, 13660-13668 (2009).

25. McNeill, E. et al. Regulation of iNOS function and cellular redox state by macrophage Gch1 reveals specific requirements for tetrahydrobiopterin in NRF2 activation. Free Radic. Biol. Med. 79C, 206-216 (2014).

26. Eckelt, E. et al. FurA contributes to the oxidative stress response regulation of Mycobacterium avium ssp. paratuberculosis. Front. Microbiol. 6, 16 (2015).

27. Humphreys, I. R. et al. A role for dendritic cells in the dissemination of mycobacterial infection. Microbes Infect. 8, 1339-1346 (2006).

28. Chuaiphichai, S. et al. Cell-autonomous role of endothelial GTP cyclohydrolase 1 and tetrahydrobiopterin in blood pressure regulation. Hypertension 64, 530-540 (2014).

29. Ajuebor, M. N., Virag, L., Flower, R. J., Perretti, M. \& Szabo, C. Role of inducible nitric oxide synthase in the regulation of neutrophil migration in zymosan-induced inflammation. Immunology 95, 625-630 (1998).

30. Merico, D., Isserlin, R., Stueker, O., Emili, A. \& Bader, G. D. Enrichment map: a network-based method for gene-set enrichment visualization and interpretation. PLoS One 5, e13984 (2010).

31. Knight, M., Braverman, J., Asfaha, K., Gronert, K. \& Stanley, S. Lipid droplet formation in Mycobacterium tuberculosis infected macrophages requires IFNgamma/HIF-1alpha signaling and supports host defense. PLoS Pathog. 14, e1006874 (2018).

32. Zelmer, A. et al. A new tool for tuberculosis vaccine screening: ex vivo mycobacterial growth inhibition assay indicates BCG-mediated protection in a murine model of tuberculosis. BMC Infect. Dis. 16, 412 (2016).

33. Tameris, M. D. et al. Safety and efficacy of MVA85A, a new tuberculosis vaccine, in infants previously vaccinated with BCG: a randomised, placebocontrolled phase $2 \mathrm{~b}$ trial. Lancet 381, 1021-1028 (2013).

34. Mishra, B. B. et al. Nitric oxide prevents a pathogen-permissive granulocytic inflammation during tuberculosis. Nat. Microbiol. 2, 17072 (2017).

35. Braverman, J., Sogi, K. M., Benjamin, D., Nomura, D. K. \& Stanley, S. A. HIFlalpha is an essential mediator of IFN-gamma-dependent immunity to Mycobacterium tuberculosis. J. Immunol. 197, 1287-1297 (2016).

36. Murray, P. J. \& Wynn, T. A. Obstacles and opportunities for understanding macrophage polarization. J. Leukoc. Biol. 89, 557-563 (2011).

37. Agranoff, D. et al. Identification of diagnostic markers for tuberculosis by proteomic fingerprinting of serum. Lancet 368, 1012-1021 (2006).

38. Qian, Z. et al. Expression of nuclear factor, erythroid 2-like 2-mediated genes differentiates tuberculosis. Tuberculosis 99, 56-62 (2016).

39. Berry, M. P. et al. An interferon-inducible neutrophil-driven blood transcriptional signature in human tuberculosis. Nature 466, 973-977 (2010).

40. Jung, J. Y. et al. The intracellular environment of human macrophages that produce nitric oxide promotes growth of mycobacteria. Infect. Immun. 81, 3198-3209 (2013).

41. Braverman, J. \& Stanley, S. A. Nitric oxide modulates macrophage responses to Mycobacterium tuberculosis infection through activation of HIF-1alpha and repression of NF-kappaB. J. Immunol. 199, 1805-1816 (2017).

42. Mishra, B. B. et al. Nitric oxide controls the immunopathology of tuberculosis by inhibiting NLRP3 inflammasome-dependent processing of IL-1beta. Nat. Immunol. 14, 52-60 (2013).

43. Correa, A. F. et al. The endothelin system has a significant role in the pathogenesis and progression of Mycobacterium tuberculosis infection. Infect. Immun. 82, 5154-5165 (2014)

44. Lam, A. et al. Role of apoptosis and autophagy in tuberculosis. Am. J. Physiol. Lung Cell. Mol. Physiol. 313, L218-L229 (2017).

45. Winkelstein, J. A. et al. Chronic granulomatous disease. Report on a national registry of 368 patients. Medicine 79, 155-169 (2000).

46. Cooper, A. M., Segal, B. H., Frank, A. A., Holland, S. M. \& Orme, I. M. Transient loss of resistance to pulmonary tuberculosis in $\mathrm{p} 47$ (phox-/-) mice. Infect. Immun. 68, 1231-1234 (2000). 
47. Pieters, J. Mycobacterium tuberculosis and the macrophage: maintaining a balance. Cell Host Microbe 3, 399-407 (2008).

48. Franco, L. H. et al. The ubiquitin ligase Smurf1 functions in selective autophagy of Mycobacterium tuberculosis and anti-tuberculous host defense. Cell Host Microbe 21, 59-72 (2017).

49. Riendeau, C. J. \& Kornfeld, H. THP-1 cell apoptosis in response to Mycobacterial infection. Infect. Immun. 71, 254-259 (2003).

50. Oddo, M. et al. Fas ligand-induced apoptosis of infected human macrophages reduces the viability of intracellular Mycobacterium tuberculosis. J. Immunol. 160, 5448-5454 (1998).

51. Shi, L. et al. Infection with Mycobacterium tuberculosis induces the Warburg effect in mouse lungs. Sci. Rep. 5, 18176 (2015).

52. Gleeson, L. E. et al. Cutting edge: Mycobacterium tuberculosis induces aerobic glycolysis in human alveolar macrophages that is required for control of intracellular bacillary replication. J. Immunol. 196, 2444-2449 (2016).

53. Mills, E. L. \& O'Neill, L. A. Reprogramming mitochondrial metabolism in macrophages as an anti-inflammatory signal. Eur. J. Immunol. 46, 13-21 (2016).

54. Bailey, J. et al. A novel role for endothelial tetrahydrobiopterin in mitochondrial redox balance. Free Radic. Biol. Med. 104, 214-225 (2017).

55. Watschinger, K. K. et al. Tetrahydrobiopterin and alkylglycerol monooxygenase substantially alter the murine macrophage lipidome. Proc. Natl. Acad. Sci. U.S.A. 112, 2431-2436 (2015).

56. Fletcher, $\mathrm{H}$. A. et al. T-cell activation is an immune correlate of risk in BCG vaccinated infants. Nat. Commun. 7, 11290 (2016).

57. Laubach, V. E., Shesely, E. G., Smithies, O. \& Sherman, P. A. Mice lacking inducible nitric oxide synthase are not resistant to lipopolysaccharide-induced death. Proc. Natl. Acad. Sci. U.S.A. 92, 10688-10692 (1995).

58. Hobbs, J. A. et al. Myeloid cell function in MRP-14 (S100A9) null mice. Mol. Cell. Biol. 23, 2564-2576 (2003).

59. Cai, S. et al. GTP cyclohydrolase I gene transfer augments intracellular tetrahydrobiopterin in human endothelial cells: effects on nitric oxide synthase activity, protein levels and dimerization. Cardiovasc. Res. 55, 838-849 (2002).

60. Matsumiya, M. et al. Gene expression and cytokine profile correlate with mycobacterial growth in a human BCG challenge model. J. Infect. Dis. 211 1499-1509 (2015).

61. Shi, W., Oshlack, A. \& Smyth, G. K. Optimizing the noise versus bias trade-off for Illumina whole genome expression BeadChips. Nucleic Acids Res. 38, e204 (2010).

62. Ritchie, M. E. et al. limma powers differential expression analyses for RNAsequencing and microarray studies. Nucleic Acids Res. 43, e47 (2015).

63. Smyth, G. K. Linear models and empirical bayes methods for assessing differential expression in microarray experiments. Stat. Appl. Genet. Mol. Biol. 3, Article3 (2004).

64. Gentleman, R. C. et al. Bioconductor: open software development for computational biology and bioinformatics. Genome Biol. 5, R80 (2004).

65. Subramanian, A. et al. Gene set enrichment analysis: a knowledge-based approach for interpreting genome-wide expression profiles. Proc. Natl. Acad. Sci. U.S.A. 102, 15545-15550 (2005).

66. Mootha, V. K. et al. PGC-1alpha-responsive genes involved in oxidative phosphorylation are coordinately downregulated in human diabetes. Nat. Genet. 34, 267-273 (2003).

67. Reich, M. et al. GenePattern 2.0. Nat. Genet. 38, 500-501 (2006).

68. Isserlin, R., Merico, D., Voisin, V. \& Bader, G. D. Enrichment Map-a Cytoscape app to visualize and explore OMICs pathway enrichment results. F1000Research 3, 141 (2014).
69. Shannon, P. et al. Cytoscape: a software environment for integrated models of biomolecular interaction networks. Genome Res. 13, 2498-2504 (2003).

70. Hulsen, T., de Vlieg, J. \& Alkema, W. BioVenn-a web application for the comparison and visualization of biological lists using area-proportional Venn diagrams. BMC Genomics 9, 488 (2008).

\section{Acknowledgements}

This work was supported by the British Heart Foundation [RG/15/10/31485], [RG/17/10/32859] and [CH/16/1/32013], Wellcome Trust [095780/Z/11/Z] and [203141/Z/16/Z], The John Fell Fund, The Oxford University Medical Research Fund, and the National Institute for Health Research (NIHR) Oxford Biomedical Research Centre. BCG-GFP was a gift from Rajko Reljic from St. George's University of London (SGUL).

\section{Author contributions}

Conceptualization: E.M.N., E.S., H.M.S., and K.M.C. Methodology: E.M.N. and E.S. Formal analysis: H.L. and J.M. Investigation: E.M.N., E.S., M.J.C., R.H.-K., R.T., M.K.S., M.M., A.-L.K., A.B.H., and M.D. Resources: P.B., H.A.F., and D.R.G. Vizualization: E.M.N. and H.L. Writing-original draft: E.M.N., E.S., H.M.S., and K.M.C. Writingreview and editing: E.M.N., E.S., D.R.G, H.M.S., and K.M.C. Funding acquisition: E.M. N., D.R.G., H.M.S., and K.M.C.

\section{Additional information}

Supplementary Information accompanies this paper at https://doi.org/10.1038/s41467018-07714-9.

Competing interests: The authors declare no competing interests.

Reprints and permission information is available online at http://npg.nature.com/ reprintsandpermissions/

Publisher's note: Springer Nature remains neutral with regard to jurisdictional claims in published maps and institutional affiliations.

\begin{abstract}
(c) (i) Open Access This article is licensed under a Creative Commons Attribution 4.0 International License, which permits use, sharing, adaptation, distribution and reproduction in any medium or format, as long as you give appropriate credit to the original author(s) and the source, provide a link to the Creative Commons license, and indicate if changes were made. The images or other third party material in this article are included in the article's Creative Commons license, unless indicated otherwise in a credit line to the material. If material is not included in the article's Creative Commons license and your intended use is not permitted by statutory regulation or exceeds the permitted use, you will need to obtain permission directly from the copyright holder. To view a copy of this license, visit http://creativecommons.org/ licenses/by/4.0/.
\end{abstract}

(C) The Author(s) 2018 